MATHEMATICS OF COMPUTATION

Volume 75 , Number 253, Pages 281-305

S 0025-5718(05)01781-3

Article electronically published on September 9, 2005

\title{
ORTHOGONAL LAURENT POLYNOMIALS CORRESPONDING TO CERTAIN STRONG STIELTJES DISTRIBUTIONS WITH APPLICATIONS TO NUMERICAL QUADRATURES
}

\author{
C. DÍAZ-MENDOZA, P. GONZÁLEZ-VERA, M. JIMÉNEZ PAIZ, \\ AND F. CALA RODRÍGUEZ
}

\begin{abstract}
In this paper we shall be mainly concerned with sequences of orthogonal Laurent polynomials associated with a class of strong Stieltjes distributions introduced by A.S. Ranga. Algebraic properties of certain quadratures formulae exactly integrating Laurent polynomials along with an application to estimate weighted integrals on $[-1,1]$ with nearby singularities are given. Finally, numerical examples involving interpolatory rules whose nodes are zeros of orthogonal Laurent polynomials are also presented.
\end{abstract}

\section{INTRODUCTION}

Let $\phi$ be a distribution function (positive measure) defined on $(a, b),-\infty \leq a<$ $b \leq \infty$. That is, $\phi$ is a real valued, bounded nondecreasing function with infinitely many points of increase on $(a, b)$, such that all the moments,

$$
c_{k}=\int_{a}^{b} x^{k} \mathrm{~d} \phi(x),
$$

exist for $k=0,1,2, \ldots$. Elsewhere in this paper, we will sometimes refer to $\phi$ as a classical distribution. When the integrals (1.1) also exist for $k=-1,-2, \ldots$, $\phi$ will be called a strong distribution. In case $\phi$ is absolutely continuous, i.e., $\mathrm{d} \phi(x)=\omega(x) \mathrm{d} x, \omega$ will be sometimes called a strong weight function. If, in addition, $0 \leq a<b \leq \infty$, then we will be handling a strong Stieltjes distribution (SSD). Such distributions were earlier introduced in 1980 by Jones et al. in their celebrated paper A Strong Stieltjes Moment Problem [17. After this, the theory of the so-called orthogonal Laurent polynomials made their appearance [18, (see also [22, 16, 5]) and connections were established with certain kinds of continued fractions, two-point Padé approximants and new quadrature rules (see [20] for a survey). On the other hand, both orthogonal Laurent polynomials and proper orthogonal polynomials

Received by the editor November 4, 2003 and, in revised form, August 2, 2004.

2000 Mathematics Subject Classification. Primary 41A21, 30E05.

Key words and phrases. Strong Stieltjes distributions, orthogonal Laurent polynomials, quadrature formulas, Stieltjes transform, two-point Padé approximants.

The first three authors were partially supported by the Scientific Research Projects of the Ministerio de Ciencia y Tecnología and Comunidad Autónoma de Canarias under contracts BFM20013411 and PI 2002/136, respectively.

The work of the fourth author was done during a visit to the Departamento de Análisis Matemático in Universidad de La Laguna (Canary Islands, Spain).

(C)2005 American Mathematical Society 
can be considered as particular cases within a more general framework: orthogonal rational functions with prescribed poles [1]. Thus, when all the poles are located at the origin and infinity Laurent polynomials arise. They are functions of the form $(p, q \in \mathbb{Z}: p \leq q)$,

$$
L(z)=\sum_{j=p}^{q} \alpha_{j} z^{j}, \quad \alpha_{j} \in \mathbb{R} .
$$

We denote by $\Delta_{p, q}$ the space of Laurent polynomials (L-polynomials) (1.2) and by $\Delta$ the space of all L-polynomials. Furthermore, for a nonnegative $k, \Pi_{k}$ will denote the space of polynomials of degree at most $k$ and $\Pi$ the space of all polynomials.

Now let $\phi$ be an SSD and consider the Hilbert space $\mathrm{L}_{2}(\mathrm{~d} \phi)$ of real functions for which $\int_{a}^{b}|f(x)|^{2} \mathrm{~d} \phi(x)<\infty, 0 \leq a<b \leq \infty$. As usual, in $\mathrm{L}_{2}(\mathrm{~d} \phi)$, we have the inner product

$$
\langle f, g\rangle_{\phi}=\langle f, g\rangle=\int_{a}^{b} f(x) g(x) \mathrm{d} \phi(x) .
$$

Next, we need to generate a sequence of nested subspaces of L-polynomials similar to the sequence $\left\{\Pi_{k}\right\}_{k \geq 0}$ of usual polynomials. For this purpose, we will start from a nondecreasing sequence $\{p(n)\}_{n \geq 0}$ of nonnegative integers such that $0 \leq p(n) \leq n$ and $s(n)=p(n)-p(n-1) \in\{0,1\}$, and set,

$$
\mathcal{L}_{n}=\Delta_{-p(n), q(n)}=\operatorname{span}\left\langle x^{j}:-p(n) \leq j \leq q(n)\right\rangle, \quad q(n)=n-p(n) .
$$

In this case it will be said that $\{p(n)\}_{n \geq 0}$, as defined above, has induced an ordering in $\mathcal{L}=\bigcup_{n \geq 0} \mathcal{L}_{n} \subset \Delta$. Observe that $\mathcal{L}_{0}=\operatorname{span}\langle 1\rangle, \operatorname{dim}\left(\mathcal{L}_{n}\right)=n+1$ and that $\mathcal{L}_{n} \subset \mathcal{L}_{n+1}, n=0,1, \ldots$. Thus, according to this ordering, a unique (up to a sign) sequence $\left\{R_{n}\right\}_{n \geq 0}$ of L-polynomials can be determined so that,

(1) $R_{n} \in \mathcal{L}_{n} \backslash \mathcal{L}_{n-1}, n=1,2, \ldots$

(2) $\left\langle R_{n}, R_{m}\right\rangle=\kappa_{n} \delta_{n, m}, \kappa_{n}>0 ; n, m=0,1, \ldots$

Setting $R_{n}(z)=\sum_{j=-p(n)}^{q(n)} r_{n, j} z^{j}$ and defining the leading coefficient of $R_{n}$ by

$$
r_{n}=\left\{\begin{array}{cc}
r_{n,-p(n)} & \text { if } s(n)=1, \\
r_{n, q(n)} & \text { if } s(n)=0,
\end{array}\right.
$$

then $r_{n} \neq 0$. If $r_{n}=1$ for each $n,\left\{R_{n}\right\}_{n \geq 0}$ will be called the monic orthogonal Lpolynomial sequence with respect to the SSD $\phi$ associated with the ordering induced by $\{p(n)\}_{n \geq 0}$. When taking $\kappa_{n}=1$, it will be called an orthonormal L-polynomial sequence denoted by $\left\{\psi_{n}\right\}_{n \geq 0}$.

The main aim of this paper is to characterize sequences of orthogonal Laurent polynomials along with their corresponding quadratures for certain SSD $\phi$ introduced by Ranga [27], i.e., $\phi$ satisfies

$$
\mathrm{d} \phi\left(\frac{\gamma^{2}}{x}\right)=-\frac{\gamma}{x} \mathrm{~d} \phi(x), \quad x \in(a, b)
$$

where $\gamma=\sqrt{a b}$, when $0<a<b<\infty$. Furthermore, if $a=0$, then $b=\infty$ and $\gamma$ can be taken as any positive arbitrary number. Such distributions are called $\gamma$-inversive. We shall also see that these distributions are related to the family of classical symmetric distributions $\mu$ on $(-d, d)(0<d \leq \infty)$; i.e., $\mu$ satisfies

$$
\mathrm{d} \mu(x)=-\mathrm{d} \mu(-x), \quad x \in(-d, d) .
$$


The paper is organized as follows. In Section 2, some properties of general sequences of orthogonal Laurent polynomials along with their associated quadrature formulas are exposed. In Section 3 these properties are considered when dealing with a $\gamma$-inversive SSD. A useful connection between the orthogonal polynomials and the Gaussian formulas for a symmetric distribution $\mu$ and certain sequences of orthogonal Laurent polynomials and their respective quadratures for the corresponding $\gamma$-inversive SSD $\phi$ will be made in Section 4. In Section 5 some illustrative examples are given. Applications of these quadrature formulas and the so-called interpolatory rules for approximating certain weighted integrals on $[-1,1]$ exhibiting singularities near the interval are given in Sections 6 and 7, respectively. Finally, some concluding remarks are provided in Section 8.

\section{Preliminary Results}

In order to make the paper self-contained, in this section some relevant properties of general sequences of orthogonal Laurent polynomials will be briefly summarized (see the recent paper 8 for details). Throughout this section $\phi$ will represent an SSD and $\left\{\mathcal{L}_{n}\right\}_{n \geq 0}$ a nested sequence of L-polynomial spaces induced by a given sequence $\{p(n)\}_{n \geq 0}$.

Concerning the zeros of $R_{n}$, there holds $([]$ ) the following theorem.

Theorem 2.1 (Zeros). For each $n$, let $R_{n}$ be an $n$th orthogonal L-polynomial for an $S S D \phi$. Then, $R_{n}$ has exactly $n$ distinct zeros on $(a, b), 0 \leq a<b \leq \infty$. Furthermore, suppose $0<x_{1, n}<x_{2, n}<\cdots<x_{n, n}$ are the zeros of $R_{n}, n=1,2, \ldots$. Then

$$
x_{j, n}<x_{j, n-1}<x_{j+1, n}, \quad j=1, \ldots, n-1, n \geq 2 .
$$

From this theorem one sees that if we write $R_{n}(z)=\sum_{j=-p(n)}^{q(n)} r_{n, j} z^{j}$, then it holds that $r_{n,-p(n)} r_{n, q(n)} \neq 0$. Throughout the paper we will make use of the normalization $r_{n, q(n)}=1$ for each $n \geq 0$, so that the sequence $\left\{R_{n}\right\}_{n \geq 0}$ will be called quasi-monic. The corresponding $n$th L-orthonormal polynomial $\bar{\psi}_{n}$ is given by $\psi_{n}=\gamma_{n} R_{n}$ with $\gamma_{n}^{2}=\frac{1}{\left\langle R_{n}, R_{n}\right\rangle}$.

As in the polynomial situation, the orthonormal sequence $\left\{\psi_{n}\right\}_{n \geq 0}$ satisfies the following $([8])$.

Theorem 2.2 (Three-term recurrence relation). Let $\left\{\psi_{n}\right\}_{n \geq 0}$ be the sequence of orthonormal L-polynomials for an $S S D \phi$. Let $s(n)=p(n)-p(n-1) \in\{0,1\}$. Then there exist two sequences of positive real numbers, $\left\{\Omega_{n}\right\}_{n \geq 1}$ and $\left\{C_{n}\right\}_{n \geq 0}$, such that for $n \geq 1$

$$
C_{n} z^{s(n+1)} \psi_{n+1}(z)=(-1)^{s(n)}\left[z^{1-2 s(n)}-\Omega_{n}\right] z^{s(n)} \psi_{n}(z)-C_{n-1} z^{s(n-1)} \psi_{n-1}(z),
$$

where

$$
C_{n}=\left([1-s(n)]-s(n) \frac{r_{n,-p(n)}}{r_{n+1,-p(n+1)}}\right) \frac{\gamma_{n}}{\gamma_{n+1}}
$$


and

$$
\begin{aligned}
\Omega_{n}= & C_{n} \frac{\gamma_{n+1}}{\gamma_{n}}\left(-\frac{r_{n+1,-p(n+1)}}{r_{n,-p(n)}}\right)^{1-s(n)} \\
& -[1-(s(n)+s(n-1))] C_{n-1} \frac{\gamma_{n-1}}{\gamma_{n}}\left(\frac{r_{n-1,-p(n-1)}}{r_{n,-p(n)}}\right)^{1-s(n)} .
\end{aligned}
$$

Formula (2.1) generalizes some known results about orthogonal polynomials and orthogonal L-polynomials. Indeed, setting $p(n)=0$, then $\mathcal{L}_{n}=\Pi_{n}$ and $s(n)=0$; hence (2.1) gives

$$
C_{n} \psi_{n+1}(z)=\left(z-\Omega_{n}\right) \psi_{n}(z)-C_{n-1} \psi_{n-1}(z),
$$

so that the well known three-term recurrence relation for orthonormal polynomials is now recovered. On the other hand, take $p(n)=\mathrm{E}[(n+1) / 2](\mathrm{E}[x]$ stands for the integer part of $x$ ), so that there holds $s(n)=0$ if $n$ is even and $s(n)=1$, otherwise. Now (2.1) yields

$$
C_{n} \psi_{n+1}(z)=(-1)^{n}\left(1-z^{(-1)^{n+1}} \Omega_{n}\right) \psi_{n}(z)-C_{n-1} \psi_{n-1}(z) .
$$

By writing $\psi_{n}(z)=Q_{n}(z) z^{-p(n)}, Q_{n} \in \Pi_{n}$, one has

$$
Q_{n+1}=\alpha_{n}\left(z-\beta_{n}\right) Q_{n}(z)-\omega_{n} z Q_{n-1}(z) .
$$

Formula (2.4) was earlier deduced in [18] and [19] (see also [5]). On the other hand, sequences of polynomials $\left\{Q_{n}\right\}_{n \geq 0}$ satisfying (2.5) have been studied extensively by Ranga et al. [26, 27, 28, 29, 31.

Assume now that the integral

$$
I_{\phi}(f)=\int_{a}^{b} f(x) \mathrm{d} \phi(x), \quad 0 \leq a<b \leq \infty,
$$

is required to be computed, and suppose that an $n$-point quadrature rule with distinct nodes $\left\{x_{j}\right\}_{j=1}^{n}$ on $(a, b)$ is used, i.e., an expression of the form,

$$
I_{n}(f)=\sum_{j=1}^{n} \lambda_{j} f\left(x_{j}\right)
$$

Then, when taking as nodes the zeros of $R_{n}$, one has ([8]) the following.

Theorem 2.3 (L-orthogonal quadratures). Let $x_{1, n}, \ldots, x_{n, n}$ be the $n$ distinct zeros of $R_{n}$. Then, there exist positive numbers $\lambda_{1, n}, \ldots, \lambda_{n, n}$ such that

$$
I_{n}(f)=\sum_{j=1}^{n} \lambda_{j, n} f\left(x_{j, n}\right)=I_{\phi}(f), \forall f \in \mathcal{L}_{n} \cdot \mathcal{L}_{n-1}=\Delta_{-(p(n)+p(n-1)), q(n)+q(n-1)},
$$

with $q(n)+p(n)=n, n=1,2, \ldots$

Remark 2.4. The quadrature formula (2.8) will be called an $n$-point L-orthogonal formula for the SSD $\phi$ and the ordering induced by $\{p(n)\}_{n \geq 0}$, and they are optimal in the sense that there cannot exist $n$-point quadrature rules (2.7) to be exact in $\mathcal{L}_{n} \mathcal{L}_{n}$. Observe that, in general, $\mathcal{L}_{n} \mathcal{L}_{n-1} \neq \mathcal{L}_{2 n-1}$, so that the L-orthogonal quadrature formulas do not coincide generally with the so-called L-Gaussian ones, which are obtained by imposing that $I_{\phi}(f)=I_{n}(f), \forall f \in \mathcal{L}_{2 n-1}$. They coincide, if and only if, $\{p(n)\}_{n \geq 0}$ satisfies $p(n)+p(n-1)=p(2 n-1)$. For example, for the above choices, $p(n)=0$ and $p(n)=\mathrm{E}[(n+1) / 2]$, this is true. 
As for the weights $\left\{\lambda_{j, n}\right\}_{j=1}^{n}$ in (2.8), we have ([8]) the following.

Theorem 2.5. Let $\psi_{n}$ be the nth of orthonormal L-polynomials for the SSD $\phi$. Then,

$$
\lambda_{j, n}=\frac{1}{C_{n-1} x_{j, n}^{s(n)+s(n-1)} \psi_{n}^{\prime}\left(x_{j, n}\right) \psi_{n-1}\left(x_{j, n}\right)}, \quad j=1, \ldots, n,
$$

$\left\{x_{j, n}\right\}_{j=1}^{n}$ being the zeros of $\psi_{n}, s(n)=p(n)-p(n-1)$ and $C_{n}$ given by (2.2).

To conclude this section, it should be remarked that the effectiveness and numerical power of the quadrature rules (2.7) in order to estimate integrals like (2.6) have been displayed recently in [2, 8. On the other hand, from the above theorems one sees that the basis to compute L-orthogonal formulas is the knowledge of the sequence $\left\{\psi_{n}\right\}_{n \geq 0}$. So it would be desirable to know explicitly (or reasonably computable, at least) the coefficients $\Omega_{n}$ and $C_{n}$ in the three-term recurrence relation (2.1). This will be done in the Section 4, taking advantage of known results about orthogonal polynomials with respect to a wide family of classical distributions.

\section{Orthogonal Laurent polynomials Associated With a CERTAin STRONG Stieltues Distribution}

Our aim in this section is to provide the characterization of sequences of orthogonal Laurent polynomial associated with a SSD $\phi$ satisfying

$$
\mathrm{d} \phi\left(\frac{\gamma^{2}}{x}\right)=-\frac{\gamma}{x} \mathrm{~d} \phi(x), \quad x \in(a, b),
$$

where $\gamma=\sqrt{a b}$ when $0<a<b<\infty$ or $\gamma$ is any positive number when $a=0$ and $b=\infty$. Such distributions are called $\gamma$-inversive and were introduced in [27] (see also [26, 28]). There, certain sequences of varying orthogonal polynomials, i.e., orthogonal polynomials with respect to the varying distributions $\mathrm{d} \phi(x) / x^{n}$, were studied emphasizing their connection with a class of continued fractions (Mfractions). In this paper, we take a different approach: since we are dealing with a strong distribution, it seams more natural to start by studying sequences of orthogonal Laurent polynomials.

As studied in [7] and [27], the influence of the $\gamma$-inversivity of $\phi$ gives rise to the following.

Definition 3.1. An $n$-point quadrature formula $I_{n}(f)=\sum_{j=1}^{n} \lambda_{j} f\left(x_{j}\right)$ for $I_{\phi}(f)$ is said to be $\gamma$-inversive, if and only if

$$
\begin{aligned}
& x_{j}=\frac{\gamma^{2}}{x_{n+1-j}}, \\
& \frac{\lambda_{j}}{\sqrt{x_{j}}}=\frac{\lambda_{n+1-j}}{\sqrt{x_{n+1-j}}}, \quad j=1, \ldots \mathrm{E}\left[\frac{n+1}{2}\right] .
\end{aligned}
$$

Now, concerning L-orthogonal quadrature formulas associated with a sequence $\{p(n)\}$ for $\phi \gamma$-inversive, we can prove the following.

Theorem 3.2. Let $I_{n}(f)=\sum_{k=1}^{n} \lambda_{k, n} f\left(x_{k, n}\right)$ be the $n$th L-orthogonal quadrature formula associated with a $\gamma$-inversive SSD $\phi$. Then $I_{n}(f)$ is $\gamma$-inversive if and only if $p(n)=\mathrm{E}[(n+1) / 2]$. 
Proof. By [7. Corollary 3.6], it holds that $I_{n}(f)$ is $\gamma$-inversive if and only if

$$
\begin{aligned}
& p(n+1)+p(n)=n+1, \quad n \geq 0, \\
& p(0)=0 .
\end{aligned}
$$

Clearly, the only solution of this first order difference equation with constant coefficients is

$$
p(n)=\frac{1}{4}\left(1+(-1)^{n+1}\right)+\frac{n}{2},
$$

which can be expressed in a closed form as

$$
p(n)=\mathrm{E}\left[\frac{n+1}{2}\right] .
$$

Remark 3.3. From this theorem one of the most commonly used orderings in the literature on orthogonal L-polynomials appears (see, e.g., [20]).

Next let us see how the property of $\gamma$-inversivity is now reflected on the sequence of orthogonal L-polynomials corresponding to $\{p(n)\}_{n \geq 0}$, such that $p(n)=\mathrm{E}\left[\frac{n+1}{2}\right]$.

Theorem 3.4. Let $\phi$ be a $\gamma$-inversive SSD and let $R_{n}$ be the nth quasi-monic orthogonal L-polynomial associated with the sequence $\{p(n)\}_{n \geq 0}$, such that $p(n)=$ $\mathrm{E}\left[\frac{n+1}{2}\right]$. Then,

$$
R_{n}(x)=\left(-\frac{\gamma}{x}\right)^{s(n)} R_{n}\left(\frac{\gamma^{2}}{x}\right) .
$$

Proof. One knows that $\int_{a}^{b} L(t) R_{n}(t) \mathrm{d} \phi(t)=0, L \in \mathcal{L}_{n-1}$. Making the change of variables $t=\frac{\gamma^{2}}{x}$, it follows that

$$
\int_{a}^{b} L\left(\frac{\gamma^{2}}{x}\right) R_{n}\left(\frac{\gamma^{2}}{x}\right) \mathrm{d} \phi\left(\frac{\gamma^{2}}{x}\right)=0, \quad L \in \mathcal{L}_{n-1},
$$

and by $\gamma$-inversibility of $\phi$,

$$
\int_{a}^{b} L\left(\frac{\gamma^{2}}{x}\right) R_{n}\left(\frac{\gamma^{2}}{x}\right) \frac{\gamma}{x} \mathrm{~d} \phi(x)=0, \quad L \in \mathcal{L}_{n-1} .
$$

Since, $R_{n}\left(\frac{\gamma^{2}}{x}\right) \in \Delta_{-q(n), p(n)}, q(n)=p(n-1), p(n)=q(n-1)+1$, and $q(n)+s(n)=$ $p(n)$, it follows that

$$
\frac{1}{x^{s(n)}} R_{n}\left(\frac{\gamma^{2}}{x}\right) \in \mathcal{L}_{n}
$$

Hence,

$$
\int_{a}^{b} L\left(\frac{\gamma^{2}}{x}\right) \frac{1}{x^{1-s(n)}} \frac{1}{x^{s(n)}} R_{n}\left(\frac{\gamma^{2}}{x}\right) \mathrm{d} \phi(x)=0, \quad L \in \mathcal{L}_{n-1}
$$

Now,

$$
\begin{aligned}
L \in \mathcal{L}_{n-1}=\Delta_{-p(n-1), q(n-1)} & \Leftrightarrow L\left(\frac{\gamma^{2}}{x}\right) \in \Delta_{-q(n-1), p(n-1)} \\
& \Leftrightarrow L\left(\frac{\gamma^{2}}{x}\right) \frac{1}{x^{1-s(n)}} \in \Delta_{-\widetilde{m}, \widehat{m}},
\end{aligned}
$$

where $\widetilde{m}=q(n-1)+1-s(n)$ and $\widehat{m}=p(n-1)-1+s(n)$. 
Taking into account that $p(n)+q(n)=n, q(n)=[1-s(n)]+q(n-1)$, and that by hypothesis $p(n)+p(n-1)=n$, it follows that $\widetilde{m}=q(n)=p(n-1)$ and $\widehat{m}=p(n)-1=q(n-1)$. Therefore,

$$
L\left(\frac{\gamma^{2}}{x}\right) \frac{1}{x^{1-s(n)}} \in \mathcal{L}_{n-1} \Leftrightarrow L \in \mathcal{L}_{n-1}
$$

yielding

$$
\frac{1}{x^{s(n)}} R_{n}\left(\frac{\gamma^{2}}{x}\right) \perp \mathcal{L}_{n-1}
$$

with respect to $\mathrm{d} \phi(x)$.

In a similar way, since the zeros of $R_{n}$ are positive, it can be proved that

$$
\frac{1}{x^{s(n)}} R_{n}\left(\frac{\gamma^{2}}{x}\right) \in \mathcal{L}_{n} \backslash \mathcal{L}_{n-1},
$$

implying that

$$
R_{n}(x)=\frac{\Delta_{n}}{x^{s(n)}} R_{n}\left(\frac{\gamma^{2}}{x}\right),
$$

where $\Delta_{n}$ is an appropriate nonzero constant.

Finally, setting $R_{n}(z)=\sum_{j=-p(n)}^{q(n)} r_{n, j} z^{j}$, and taking into account that $r_{n, q(n)}=$ 1 and, hence, $r_{n,-p(n)}=(-1)^{n} \prod_{j=1}^{n} x_{j, n}$, it can be seen by (3.1) that

$$
r_{n,-p(n)}=(-\gamma)^{n} \text {. }
$$

Hence, from (3.6) and again recalling that $p(n)+p(n-1)=n$, one deduces that $\Delta_{n}=(-\gamma)^{s(n)}$ and the proof is concluded.

Reciprocally,

Theorem 3.5. Let $\left\{R_{n}\right\}_{n \geq 0}$ be the sequence of quasi-monic orthogonal Laurent polynomials for a the $\gamma$-inversive $S S D \phi$ and the ordering induced by $\{p(n)\}_{n \geq 0}$. If

$$
R_{n}(x)=\left(-\frac{\gamma}{x}\right)^{s(n)} R_{n}\left(\frac{\gamma^{2}}{x}\right),
$$

then $p(n)=\mathrm{E}\left[\frac{n+1}{2}\right], n=0,1, \ldots$

Proof. Proceeding similarly as done in the proof of Theorem 3.4, from (3.4)-(3.5) one deduces that

$$
R_{n} \perp \Delta_{-\widetilde{m}, \widehat{m}},
$$

where $\widetilde{m}=q(n-1)+1-s(n)$ and $\widehat{m}=p(n-1)-1+s(n)$. Furthermore, $\widetilde{m} \leq p(n-1)$ and $\widehat{m} \leq q(n-1)$ since, otherwise, $R_{n} \equiv 0$ because it satisfies $n+1$ orthogonality conditions. Assume that $\widetilde{m}<p(n-1)$, i.e., $q(n-1)+1-s(n)<p(n-1)$ or equivalently, $q(n-1)<p(n-1)-1+s(n)$. Since $p(n-1)-1+s(n) \leq q(n-1)$, a contradiction appears. Therefore, there holds $q(n-1)+1-s(n)=p(n-1)$.

In a similar way, if we assume that $p(n-1)-1+s(n)<q(n-1)$, one deduces that $p(n-1)<p(n-1)$. Hence, $p(n-1)-1+s(n)=q(n-1)$.

Now, taking into account that $p(n-1)+q(n-1)=n-1$ and $p(n)=p(n-1)+s(n)$, it follows that $p(n)+p(n-1)=n$ with $p(0)=0$, and the proof is concluded.

Let us next see how relation (2.1) can now be reformulated. By Theorem 2.2 . one has: 
Theorem 3.6. Let $\left\{R_{n}\right\}_{n \geq 0}$ be the sequence of quasi-monic orthogonal L-polynomials for a $\gamma$-inversive $S S D \phi$ with respect to an ordering induced by $\{p(n)\}_{n \geq 0}$, with $p(n)=\mathrm{E}\left[\frac{n+1}{2}\right]$. Then,

$$
R_{n+1}(z)=(z-\gamma) z^{s(n)-1} R_{n}(z)-\left(\frac{\gamma_{n-1}}{\gamma_{n}}\right)^{2} \gamma^{2 s(n)-1} R_{n-1}(z),
$$

where $\gamma_{n}^{2}=\frac{1}{\left\langle R_{n}, R_{n}\right\rangle}$ and $s(n)=p(n)-p(n-1)$.

Proof. By Theorem 2.2, one has

$$
C_{n} x^{s(n+1)} \psi_{n+1}(x)=(-1)^{s(n)}\left(x^{1-2 s(n)}-\Omega_{n}\right) x^{s(n)} \psi_{n}(x)-C_{n-1} x^{s(n-1)} \psi_{n-1}(x),
$$

with $C_{n}$ and $\Omega_{n}$ given by (2.2) and (2.3), respectively. Take into account that now $s(n)+s(n-1)=1$ and that by (3.7) it can be deduced that $\Omega_{n}=\gamma^{1-2 s(n)}$ and $C_{n}=\frac{\gamma_{n}}{\gamma_{n+1}} \gamma^{-s(n)}$. Since $\psi_{n}=\gamma_{n} R_{n}$, it follows that

$$
\begin{aligned}
C_{n} \gamma_{n+1} x^{s(n+1)} R_{n+1}(x)= & (-1)^{s(n)}\left[x^{1-2 s(n)}-\gamma^{1-2 s(n)}\right] \gamma_{n} x^{s(n)} R_{n}(x) \\
& -C_{n-1} \gamma_{n-1} x^{s(n-1)} R_{n-1}(x) .
\end{aligned}
$$

Making $x=\frac{\gamma^{2}}{z}$ by (3.3), one can write

$$
\begin{aligned}
C_{n} \gamma_{n+1} & (-\gamma)^{s(n+1)} R_{n+1}(z) \\
= & (-1)^{s(n)}\left[\left(\frac{\gamma^{2}}{z}\right)^{1-2 s(n)}-\gamma^{1-2 s(n)}\right] \gamma_{n}(-\gamma)^{s(n)} R_{n}(z) \\
& -C_{n-1} \gamma_{n-1}(-\gamma)^{s(n-1)} R_{n-1}(z),
\end{aligned}
$$

or equivalently,

$$
\begin{aligned}
R_{n+1}(z)= & (-1)^{s(n)}\left[\left(\frac{\gamma^{2}}{z}\right)^{1-2 s(n)}-\gamma^{1-2 s(n)}\right] \frac{\gamma_{n}}{C_{n} \gamma_{n+1}(-\gamma)^{s(n+1)-s(n)}} R_{n}(z) \\
& -\frac{C_{n-1} \gamma_{n-1}}{C_{n} \gamma_{n+1}} R_{n-1}(z),
\end{aligned}
$$

since $s(n+1)=s(n-1)\left(\right.$ recall that $s(n)=p(n)-p(n-1)$ and that $\left.p(n)=\left[\frac{n+1}{2}\right]\right)$.

Now, since $C_{n}=\frac{\gamma_{n}}{\gamma_{n+1}} \gamma^{-s(n)}$, one deduces that

$$
\frac{\gamma_{n}}{C_{n} \gamma_{n+1}(-\gamma)^{s(n+1)-s(n)}}=-\left[(1-s(n)) \frac{1}{\gamma}+s(n) \gamma^{2}\right]
$$

and that

$$
\frac{C_{n-1} \gamma_{n-1}}{C_{n} \gamma_{n+1}}=\left(\frac{\gamma_{n-1}}{\gamma_{n}}\right)^{2} \gamma^{2 s(n)-1}
$$

Finally, by considering that $s(n) \in\{0,1\}$, or more precisely $s(n)=0$ if $n$ is even and $s(n)=1$, otherwise, the proof follows.

\section{A COnNeCtion With Classical Symmetric Distributions}

In general and according to the results shown in Section 2, the calculation of the L-orthogonal quadrature formulas (2.8) basically depend on the computation of the sequence $\left\{R_{n}\right\}_{n \geq 0}$ (or $\left\{\psi_{n}\right\}_{n \geq 0}$ ) by means of the three-term recurrence relation (2.1). Thus, the recurrence coefficients $C_{n}$ and $\Omega_{n}$ should be efficiently computed (see, e.g., 9] for the polynomial situation). 
However, as seen throughout Section 3, when dealing with a $\gamma$-inversive SSD, the recursion coefficients reduce to $\gamma_{n}^{2}=\frac{1}{\left\langle R_{n}, R_{n}\right\rangle}$ and computations are extremely well facilitated.

On the other hand, from (3.1) and (3.2) for a $\gamma$-inversive SSD only half of the weights and nodes needs to be computed. Furthermore, because of the intimate connection between the $\gamma$-inversive SSD and the classical symmetric distribution, all the involved computations are going to be greatly simplified. This is the aim of the present section.

We denote by $\mu$ a symmetric distribution on $(-d, d)$. To fix ideas and for the sake of simplicity, we will assume that $\mu$ is absolutely continuous; i.e.,

$$
\mathrm{d} \mu(t)=\sigma(t) \mathrm{d} t, \quad \text { such that } \quad \sigma(-t)=\sigma(t) \quad \forall t \in(-d, d)
$$

( $\sigma$ is a weight function, i.e., $\sigma(t)>0$ a.e. on $(-d, d)$ ).

Now, according to the ideas given in [13] (see also [14, 15], along with [28, for an alternative approach), where a general procedure to obtain strong distributions is provided, let us consider the transformation

$$
t=V(x)=\frac{1}{\delta}\left(x-\frac{\gamma}{x}\right)
$$

with $\delta$ and $\gamma$ given real positive numbers.

Thus, when $d<\infty$, (4.2) maps $B=[-\sqrt{b},-\sqrt{a}] \cup[\sqrt{a}, \sqrt{b}]$ on $[-d, d]$ by choosing $\delta$ and $\gamma$ such that

$$
\delta=\frac{\sqrt{b}-\sqrt{a}}{d}, \quad \gamma=\sqrt{a b},
$$

where $a$ and $b$ are positive numbers with $a<b$. On the other hand, when $d=\infty$, then for any pair $(\delta, \gamma)$ of positive numbers, (4.2) maps $(-\infty, 0) \cup(0, \infty)$ onto $\mathbb{R}$ (observe that $V(-x)=-V(x)$ ).

Now let us take the distribution,

$$
\mathrm{d} \phi(x)=\frac{1}{\sqrt{x}} \sigma(V(\sqrt{x})) \mathrm{d} x=\omega(x) \mathrm{d} x .
$$

Then it can be easily checked that $\phi$ is a $\gamma$-inversive $\mathrm{SSD}$ on $(a, b), 0 \leq a<b \leq \infty$. Furthermore, from the considerations above, it holds that $d=\infty$ if and only if $a=0$ and $b=\infty$.

Now consider the ordering in $\mathcal{L}$ induced by the sequence $\{p(n)\}_{n \geq 0}$ such that $p(n)=\mathrm{E}[(n+1) / 2], n=0,1,2, \ldots$, let $\left\{R_{n}\right\}_{n \geq 0}$ denote the corresponding sequence of quasi-monic orthogonal L-polynomials for the SSD (4.4), and let $\left\{P_{n}\right\}_{n \geq 0}$ be the sequence of monic orthogonal polynomials for $\sigma$ satisfying (4.1). Under these considerations, we have the following.

Proposition 4.1. It holds that $R_{n}(x)=x^{-\frac{s(n)}{2}} \delta^{n} P_{n}(V(\sqrt{x}))$ for $n=0,1,2, \ldots$

Proof. Set $Q_{2 n}(x)=\delta^{n} P_{n}(V(x))$. Then, by [15, Theorem 2.2.9], $Q_{2 n}$ represents the $2 n$th quasi-monic orthogonal L-polynomial with respect to $\widetilde{\omega}(x)=\sigma(V(x)), x \in B$, and the ordering induced by $p(n)=\mathrm{E}[(n+1) / 2](p(n-1)+p(n)=p(2 n-1)=n)$; i.e., $Q_{2 n} \in \mathcal{L}_{2 n}=\Delta_{-n, n}$, so that $Q_{2 n}(x)=\sum_{j=-n}^{n} C_{2 n, j} x^{j}\left(C_{2 n, n}=1\right)$ and

$$
\int_{B} R(x) Q_{2 n}(x) \widetilde{\omega}(x) \mathrm{d} x=0, \quad \forall R \in \mathcal{L}_{2 n-1}=\Delta_{-n, n-1} .
$$


Here, $B=[-\sqrt{b},-\sqrt{a}] \cup[\sqrt{a}, \sqrt{b}]$, with $a$ and $b$ satisfying (4.3) if $d$ is finite or $B=(-\infty, 0) \cup(0, \infty)$ if $d=\infty$. Taking into account the symmetry of the domain $B(B=-B)$ and the symmetry of the weight function $(\widetilde{\omega}(x)=\widetilde{\omega}(-x))$, it follows that

$$
Q_{2 n}(x)=(-1)^{n} Q_{2 n}(-x) .
$$

Hence, it can be written

$$
Q_{2 n}(x)=x^{s(n)} N_{n}\left(x^{2}\right), \quad N_{n} \in \mathcal{L}_{n} \backslash \mathcal{L}_{n-1} .
$$

Thus, (4.5) can be now expressed as

$$
\int_{B} R(x) x^{s(n)} N_{n}\left(x^{2}\right) \widetilde{\omega}(x) \mathrm{d} x=0, \quad \forall R \in \mathcal{L}_{2 n-1},
$$

or, equivalently, as

$$
\int_{B} L\left(x^{2}\right) N_{n}\left(x^{2}\right) \widetilde{\omega}(x) \mathrm{d} x=0, \quad \forall L \in \mathcal{L}_{n-1} .
$$

Indeed,

$$
\begin{aligned}
R \in \mathcal{L}_{2 n-1} & =\Delta_{-n, n-1}=\Delta_{-(p(n)+p(n-1)),(2 q(n-1)+1-s(n))} \\
& \Leftrightarrow x^{s(n)} R(x) \in \Delta_{-2 p(n-1), 2 q(n-1)+1},
\end{aligned}
$$

and, since $N_{n}\left(x^{2}\right) \widetilde{\omega}(x)$ is an even function, when considering the odd powers, the integral over $B$ vanishes. Setting $x^{2}=t$ in (4.6), one deduces

$$
\int_{a}^{b} L(t) N_{n}(t) \omega(t) \mathrm{d} t=0, \quad \forall L \in \mathcal{L}_{n-1}
$$

Since $N_{n}$ is also quasi-monic there holds

$$
R_{n}(x)=N_{n}(x)=x^{-\frac{s(n)}{2}} Q_{2 n}(\sqrt{x})=x^{-\frac{s(n)}{2}} \delta^{n} P_{n}(V(\sqrt{x})) .
$$

Now, taking into account that $t=V(\sqrt{x})=\frac{1}{\delta}\left(\sqrt{x}-\frac{\gamma}{\sqrt{x}}\right)$ represents a one-toone map between $(a, b)$ and $(-d, d)$, we have

Corollary 4.2. Let $\left\{x_{j, n}\right\}_{j=1}^{n}$ be the $n$ zeros of the $n$th quasi-monic orthogonal Lpolynomial $R_{n}$ with respect to the $\gamma$-inversive SSD (4.4) associated with the ordering induced by $\{p(n)\}_{n \geq 0}$ such that $p(n)=\mathrm{E}\left[\frac{n+1}{2}\right]$ and let $\left\{t_{j, n}\right\}_{j=1}^{n}$ the zeros of $P_{n}$ the nth monic orthogonal polynomial for $\sigma$ satisfying (4.1). Then,

$$
x_{j, n}=\left(\frac{\delta t_{j, n}+\sqrt{\left(\delta t_{j, n}\right)^{2}+4 \gamma}}{2}\right)^{2}, \quad j=1, \ldots, n .
$$

Let us next see the relation between the $\mathrm{L}_{2}$-norms of $R_{n}$ and $P_{n}$ associated with $\omega$ and $\sigma$, respectively. Indeed, by setting

$$
\Theta_{n}^{-2}=\int_{-d}^{d} P_{n}^{2}(t) \sigma(t) \mathrm{d} t
$$

and

$$
\gamma_{n}^{-2}=\int_{a}^{b} R_{n}^{2}(x) \mathrm{d} \phi(x)
$$

one has 
Proposition 4.3. Let $R_{n}$ be nth quasi-monic orthogonal Laurent polynomial associated with the $\gamma$-inversive SSD $\phi$ (4.4) and the ordering induced by $\{p(n)\}_{n \geq 0}$ such that $p(n)=\mathrm{E}\left[\frac{n+1}{2}\right]$, and let $P_{n}$ be the nth monic orthogonal polynomial with respect to the symmetric distribution $\sigma$ (4.1). Then

$$
\frac{1}{\gamma_{n}^{2}}=\frac{\delta^{2 n+1}}{\gamma^{s(n)}} \frac{1}{\Theta_{n}^{2}}
$$

where $s(n)=p(n)-p(n-1)$.

Proof. By Proposition 4.1 ,

$$
\begin{aligned}
\frac{1}{\gamma_{n}^{2}} & =\int_{a}^{b} R_{n}^{2}(x) \frac{\sigma(V(\sqrt{x}))}{\sqrt{x}} \mathrm{~d} x \\
& =\delta^{2 n} \int_{a}^{b} \frac{P_{n}^{2}(V(\sqrt{x}))}{x^{s(n)}} \frac{\sigma(V(\sqrt{x}))}{\sqrt{x}} \mathrm{~d} x .
\end{aligned}
$$

Now, since

$$
x=u(t)=\left(\frac{\delta t+\sqrt{(\delta t)^{2}+4 \gamma}}{2}\right)^{2}
$$

is the inverse transform of $t=V(\sqrt{x})$, it follows that

$$
d x=\frac{2 \delta u(t) \mathrm{d} t}{\sqrt{(\delta t)^{2}+4 \gamma}} .
$$

Therefore,

$$
\frac{1}{\gamma_{n}^{2}}=2 \delta^{2 n+1} \int_{-d}^{d} P_{n}^{2}(t) u(t)^{1-s(n)-\frac{1}{2}} \frac{\sigma(t) \mathrm{d} t}{\sqrt{(\delta t)^{2}+4 \gamma}} .
$$

If $s(n)=0$, then

$$
\frac{u(t)^{1-s(n)-\frac{1}{2}}}{\sqrt{(\delta t)^{2}+4 \gamma}}=\frac{1}{2}\left(\frac{\delta t}{\sqrt{(\delta t)^{2}+4 \gamma}}+1\right) .
$$

In this case and since $n$ is even, $P_{n}$ is an even polynomial function; hence

$$
\int_{-d}^{d} \frac{t P_{n}^{2}(t) \sigma(t)}{\sqrt{(\delta t)^{2}+4 \gamma}} \mathrm{d} t=0
$$

(recall that $\sigma$ is also even). Thus, it holds that

$$
\frac{1}{\gamma_{n}^{2}}=\frac{\delta^{2 n+1}}{\Theta_{n}^{2}}
$$

Assume now that $s(n)=1$, that is, $n$ is odd. Then

$$
\begin{aligned}
\frac{u(t)^{1-s(n)-\frac{1}{2}}}{\sqrt{(\delta t)^{2}+4 \gamma}} & =\frac{1}{2}\left(\frac{\delta t-\sqrt{(\delta t)^{2}+4 \gamma}}{-\gamma \sqrt{(\delta t)^{2}+4 \gamma}}\right) \\
& =\frac{1}{2 \gamma}-\frac{\delta t}{2 \gamma \sqrt{(\delta t)^{2}+4 \gamma}} .
\end{aligned}
$$

Here we have made use of the fact that

$$
\left(\frac{\delta t+\sqrt{(\delta t)^{2}+4 \gamma}}{2}\right)\left(\frac{\delta t-\sqrt{(\delta t)^{2}+4 \gamma}}{2}\right)=-\gamma .
$$


Now $P_{n}$ is an odd polynomial function. So, proceeding as above, one deduces that

$$
\frac{1}{\gamma_{n}^{2}}=\frac{\delta^{2 n+1}}{\gamma} \frac{1}{\Theta_{n}^{2}}
$$

Remark 4.4. From Proposition 4.3, the three-term recurrence relation (3.8) now reads

$$
R_{n+1}(x)=(x-\gamma) x^{s(n)-1} R_{n}(x)-\delta^{2} \frac{\Theta_{n-1}^{2}}{\Theta_{n}^{2}} R_{n-1}(x),
$$

with $\Theta_{n}$ given by (4.8) and, as usual, $s(n)=0$ when $n$ is even and $s(n)=1$, otherwise. Furthermore, by Proposition 4.1 and (4.10), the well known recurrence relation for the sequence $\left\{P_{n}\right\}_{n \geq 0}$ is recovered. Namely,

$$
P_{n+1}(t)=t P_{n}(t)-a_{n}^{2} P_{n-1}(t)
$$

with

$$
a_{n}^{2}=\frac{\int_{-d}^{d} P_{n}^{2}(t) \sigma(t) \mathrm{d} t}{\int_{-d}^{d} P_{n-1}^{2}(t) \sigma(t) \mathrm{d} t}
$$

(see 33$]$ ).

Until now, we have seen how the nodes $\left\{x_{j, n}\right\}_{j=1}^{n}$ of the $n$-point L-orthogonal quadrature formula for the $\gamma$-inversive SSD $\phi$ (4.4) can be expressed in terms of the zeros $\left\{t_{j, n}\right\}_{j=1}^{n}$ of the $n$th orthogonal polynomial with respect to $\sigma$ (4.1); i.e., in terms of the nodes of the $n$-point Gauss formula for this weight function, namely

$$
\widetilde{I}_{n}(f)=\sum_{j=1}^{n} A_{j, n} f\left(t_{j, n}\right)=\int_{-d}^{d} f(x) \sigma(x) \mathrm{d} x, \quad \forall f \in \Pi_{2 n-1},
$$

where the Christoffel numbers $\left\{A_{j, n}\right\}_{j=1}^{n}$ are given by (see [33])

$$
A_{j, n}=\frac{1}{\Theta_{n-1}^{2} P_{n}^{\prime}\left(t_{j, n}\right) P_{n-1}\left(t_{j, n}\right)}, \quad j=1, \ldots, n .
$$

In this respect, the next result allows us to express the weights $\left\{\lambda_{j, n}\right\}_{j=1}^{n}$ in terms of $\left\{A_{j, n}\right\}_{j=1}^{n}$.

Proposition 4.5. Let $\sigma$ be a symmetric weight function on $(-d, d), d>0$, and let $A_{1, n}, \ldots, A_{n, n}$ be its nth Christoffel numbers. Let $\phi$ be a $\gamma$-inversive SSD $\phi$ given by (4.4), and let $\lambda_{1, n}, \ldots, \lambda_{n, n}$, be the weights of the $n$-point L-orthogonal quadrature formula for $\phi$ associated with the ordering induced by $\{p(n)\}_{n \geq 0}$ such that $p(n)=\mathrm{E}\left[\frac{n+1}{2}\right]$. Then

$$
\lambda_{j, n}=\frac{2 \delta}{1+\frac{\gamma}{x_{j, n}}} A_{j, n}, \quad j=1, \ldots, n,
$$

where $\left\{x_{j, n}\right\}_{j=1}^{n}$ is the set of nodes of the $n$-point L-orthogonal quadrature formula.

Proof. By (2.9) in Theorem 2.5 and recalling that $s(n)+s(n-1)=1$,

$$
\begin{aligned}
\lambda_{j, n} & =\frac{1}{C_{n-1} x_{j, n}^{s(n)+s(n-1)} \psi_{n}^{\prime}\left(x_{j, n}\right) \psi_{n-1}\left(x_{j, n}\right)} \\
& =\frac{1}{C_{n-1} x_{j, n} \gamma_{n} \gamma_{n-1} R_{n}^{\prime}\left(x_{j, n}\right) R_{n-1}\left(x_{j, n}\right)} .
\end{aligned}
$$


On the other hand, $x^{\frac{s(n)}{2}} R_{n}(x)=\delta^{n} P_{n}(V(\sqrt{x}))$ with $V(x)=\frac{1}{\gamma}\left(x-\frac{\gamma}{x}\right)$, yielding

$$
x_{j, n}^{\frac{s(n)}{2}} R_{n}^{\prime}\left(x_{j, n}\right)=\delta^{n} P_{n}^{\prime}\left(V\left(\sqrt{x_{j, n}}\right)\right) \frac{V^{\prime}\left(\sqrt{x_{j, n}}\right)}{2 \sqrt{x_{j, n}}} .
$$

Furthermore, one knows that

$$
R_{n-1}\left(x_{j, n}\right)=\frac{\delta^{n-1} P_{n-1}\left(V\left(\sqrt{x_{j, n}}\right)\right)}{x_{j, n}^{\frac{s(n-1)}{2}}} .
$$

Thus, since $t_{j, n}=V\left(\sqrt{x_{j, n}}\right)$, it follows that

$$
\lambda_{j, n}=\frac{2}{C_{n-1} \gamma_{n} \gamma_{n-1} \delta^{2 n-1} P_{n}^{\prime}\left(t_{j, n}\right) P_{n-1}\left(t_{j, n}\right) V^{\prime}\left(\sqrt{x_{j, n}}\right)} .
$$

Now, from (3.9) we can calculate $C_{n-1} \gamma_{n} \gamma_{n-1}$, yielding

$$
C_{n-1} \gamma_{n} \gamma_{n-1}=-\frac{\gamma_{n-1}^{2}}{(-\gamma)^{s(n)-s(n-1)}\left[(1-s(n-1)) \frac{1}{\gamma}+s(n-1) \gamma^{2}\right]},
$$

and since by (4.9) $\gamma_{n-1}^{2}=\frac{\gamma^{s(n-1)}}{\delta^{2 n-1}} \Theta_{n-1}^{2}$, it holds that

$$
\lambda_{j, n}=\frac{2}{\Theta_{n-1}^{2} P_{n}^{\prime}\left(t_{j, n}\right) P_{n-1}\left(t_{j, n}\right) V^{\prime}\left(\sqrt{x_{j, n}}\right)} .
$$

Finally, $V^{\prime}\left(\sqrt{x_{j, n}}\right)=\frac{1}{\delta}\left(1+\frac{\gamma}{x_{j, n}}\right)$, so that from (4.11), the proof is concluded.

\section{EXAmples}

In order to illustrate the above theory and taking into account the lack in the literature of sequences of orthogonal L-polynomials similar to the known families of classical orthogonal polynomials (Chebyshev, Legendre, etc.) that can be easily handled, throughout this section we will deal with a certain family of $\gamma$-inversive SSD $\phi$ obtained from known symmetric weight functions on $(-1,1)$. In this respect, those examples considered in [27] (see also [26, 29]) like $\mathrm{d} \phi(x)=\frac{\mathrm{d} x}{\sqrt{(b-x)(x-a)}}$ and $\mathrm{d} \phi(x)=\frac{\mathrm{d} x}{\sqrt{x}}$ on $(a, b)(0<a<b<\infty)$ can now be deduced from the general procedure introduced in Section 4.

Thus, we will concentrate on the one-parameter family of $\operatorname{SSD}, \mathrm{d} \phi^{\mu}(x)=$ $\omega^{\mu}(x) \mathrm{d} x$, such that

$$
\omega^{\mu}(x)=\frac{(b-x)^{\mu-\frac{1}{2}}(x-a)^{\mu-\frac{1}{2}}}{(\sqrt{b}-\sqrt{a})^{2 \mu-1} x^{\mu}}, \quad x \in(a, b),
$$

where $0<a<b<\infty$ and $\mu>-1 / 2$. The corresponding sequence of orthogonal L-polynomials will be called of Gegenbauer-type because (5.1) corresponds with the so-called strong extension of a weight function, which gives rise to the well-known Gegenbauer or ultraspherical polynomials, namely,

$$
\sigma(t)=\left(1-t^{2}\right)^{\mu-\frac{1}{2}}, \quad t \in(-1,1), \quad \mu>-\frac{1}{2},
$$

when we consider (4.4) and (4.2)-(4.3), with $d=1$. 
Let $\left\{R_{n}^{(\mu)}\right\}_{n \geq 0}$ denote the sequence of quasi-monic orthogonal L-polynomials for (5.1). Then, making use of (4.10) one obtains the three-term recurrence relation

$$
R_{n+1}^{(\mu)}(x)=(x-\gamma) x^{s(n)-1} R_{n}^{(\mu)}(x)-\delta^{2} \frac{\Theta_{n-1}^{2}}{\Theta_{n}^{2}} R_{n-1}^{(\mu)}(x), \quad n \geq 1,
$$

where $R_{0}^{(\mu)}(x) \equiv 1, R_{1}^{(\mu)}(x)=1-\frac{\gamma}{x}$ and $\frac{\Theta_{n-1}^{2}}{\Theta_{n}^{2}}$ is now explicitly given by ([33])

$$
\frac{\Theta_{n-1}^{2}}{\Theta_{n}^{2}}=\frac{n(n+2 \mu-1)}{4(n+\mu-1)(n+\mu)}, \quad n \geq 1 .
$$

On the other hand, the study of Gaussian formulas for the ultraspherical weights $\sigma(t)=\left(1-t^{2}\right)^{\mu-1 / 2}, \mu>-1 / 2$, has received much attention throughout the development of the theory on orthogonal polynomials. One of the aims of these studies consisted of giving a representation for the Christoffel numbers in terms of the zeros of the Gegenbauer polynomials that was as simple as possible. In this respect, explicit representations were obtained (see 33]) for the cases $\mu=0,1$ and 21] for $\mu=2$. Such representations were recently generalized for $\mu=\ell$, an arbitrary nonnegative integer in 24] (see also 25] for an alternative expression). Thus, making use of these results, explicit expressions for the weights $\left\{\lambda_{j}^{(\ell)}\right\}_{j=1}^{n}, \ell=0,1,2, \ldots$ of the $n$-point L-orthogonal quadrature formula for the SSD (5.1) can now be given. Indeed, for $\ell$ a given nonnegative integer, let $\left\{t_{j}^{(\ell)}\right\}_{j=1}^{n}$ denote the zeros of the $n$th monic Gegenbauer polynomial $P_{n}^{(\ell)}(t)$ and set

$$
N=n+\ell \quad \text { and } \quad \alpha_{j}(\ell)=\left(\frac{(2 j) !}{2^{j} j !}\right)^{2}\left(\begin{array}{c}
\ell-1+j \\
2 j
\end{array}\right), \quad j=1,2, \ldots
$$

Then, making use of [24, formula (4.1)] and Proposition 4.5, the following holds for $j=1, \ldots, n, \ell=0,1,2, \ldots$ and $n \geq 1$ :

$$
\lambda_{j}^{(\ell)}=\frac{2(\sqrt{b}-\sqrt{a})}{1+\frac{\sqrt{a b}}{x_{j}^{(\ell)}}} \frac{\pi}{N}\left(1-\left(t_{j}^{(\ell)}\right)^{2}\right)^{\ell}\left(1+\sum_{k=1}^{\ell-1} \frac{\alpha_{k}(\ell)}{\left(1-\left(t_{j}^{(\ell)}\right)^{k}\right)} \prod_{r=1}^{k} \frac{1}{N^{2}-r^{2}}\right),
$$

with $N$ and $\alpha_{j}^{(\ell)}$ given by (5.2). Clearly, by assuming the convention that the summation in (5.3) is taken as zero for $\ell \in\{0,1\}$, from (5.3), the weights $\left\{\lambda_{j}^{(\ell)}\right\}_{j=1}^{n}$ $(\ell=0,1)$ corresponding to the so called strong Chebyshev distribution of the first and second kind are now directly recovered (compare with [28]):

$$
\begin{aligned}
\lambda_{j}^{(0)} & =\frac{2 \pi \delta}{n} \frac{x_{j}}{x_{j}+\gamma}, \\
\lambda_{j}^{(1)} & =\frac{2 \pi \delta}{n+1} \sin ^{2}\left(\frac{j \pi}{n+1}\right) \frac{x_{j}}{x_{j}+\gamma}, \quad j=1, \ldots, n .
\end{aligned}
$$

On the other hand, for $\ell=2$, one obtains for $j=1, \ldots, n, n \geq 1$,

$$
\lambda_{j}^{(2)}=\frac{2(\sqrt{b}-\sqrt{a})}{1+\frac{\sqrt{a b}}{x_{j}^{(2)}}} \frac{\pi}{n+2}\left(1-\left(t_{j}^{(2)}\right)^{2}\right)^{2}\left(1+\frac{1}{\left(1-t_{j}^{(2)}\right)\left(n^{2}+4 n+3\right)}\right) .
$$


Finally, the case $\ell=3$ reads as follows:

$$
\begin{aligned}
\lambda_{j}^{(3)}= & \frac{2(\sqrt{b}-\sqrt{a})}{1+\frac{\sqrt{a b}}{x_{j}^{(3)}}} \frac{\pi}{n+3}\left(1-\left(t_{j}^{(3)}\right)^{2}\right)^{3} \\
& \times\left(1+\frac{3}{(n+2)(n+4)\left(1-\left(t_{j}^{(3)}\right)^{2}\right)}\right. \\
& \left.\quad+\frac{9}{(n+1)(n+2)(n+4)(n+5)\left(1-\left(t_{j}^{(3)}\right)^{2}\right)^{3}}\right) \\
= & \left.\frac{2(\sqrt{b}-\sqrt{a})}{1+\frac{\sqrt{a b}}{x_{j}^{(3)}}} \frac{\pi}{n+3}\left(1-\left(t_{j}^{(3)}\right)^{2}\right)\right)^{3} \\
& \times\left(1+\frac{3}{(n+2)(n+4)\left(1-\left(t_{j}^{(3)}\right)^{2}\right)}\left(1+\frac{3}{(n+1)(n+5)\left(1-\left(t_{j}^{(3)}\right)^{2}\right)}\right)\right)
\end{aligned}
$$

for $j=1, \ldots, n, n \geq 1$.

\section{Approximate CAlCulation \\ OF WEIGHTED INTEGRALS WITH NEARBY SINGULARITIES}

As an application of the quadrature formulas given in Section 4, suppose we are concerned with the computation of integrals,

$$
\int_{-1}^{1} f(t) \sigma(t) \mathrm{d} t
$$

where $f$ is a continuous function on $[-1,1]$ with real singularities outside this interval and $\sigma$ is a weight function on $[-1,1]$. If we assume that $\beta$ is one of these singularities, then $|\beta|>1$ and, as can be expected, when $|\beta|$ is close to 1 , the GaussChristoffel quadrature rules for $\sigma$ should exhibit a slow convergence behaviour (as will be checked numerically below). In order to overcome this drawback, we will proceed as follows (see [27] for $\sigma(t)=\left(1-t^{2}\right)^{-1 / 2}$ and also [6]).

Consider $a$ and $b$ any two positive real numbers such that $(|\beta|+1)(|\beta|-1)^{-1}=$ $b / a$, and set the change of variables

$$
t=h(x)=-\frac{|\beta|}{\beta}\left(\frac{2 x-b-a}{b-a}\right) .
$$

So,

$$
I(f)=\int_{-1}^{1} f(t) \sigma(t) \mathrm{d} t=\left(\frac{2}{b-a}\right) \int_{a}^{b} g(x) \rho(x) \mathrm{d} x,
$$

where $\rho(x)=\sigma(h(x))$ and $g(x)=f(h(x))$ is a continuous function on $[a, b]$ with a singularity at the origin. Thus, by taking sufficiently large $a$, one might initially think of estimating the integral on the right hand side of (6.1) by means of the $n$-point Gaussian formula for $\rho(x)$. But since we are dealing with linear transformations, this is equivalent to estimating $I(f)$ by the corresponding $n$-point Gaussian formula for $\sigma(t)$, and the results are extremely poor. However and because of the presence of this singularity at the origin, it seems natural to expect somewhat good numerical results when the integral in the right hand side in (6.1) is estimated by using an L-orthogonal quadrature formula for $\rho$. 
The numerical experiments carried out come to confirm this expectation. However, the computational cost required could now be extremely high and this drastically reduces the competence of our approach. Indeed, one needs to efficiently compute an $n$-point L-orthogonal formula for the strong weight function $\rho(x)$, and this should be done from the three-term recurrence relation (2.1). As in the polynomial case, this approach could require a long computational process leading to possible instabilities and other related problems. Fortunately, we will see that these negative circumstances can be completely avoided for the particular case

$$
\sigma(t)=\left(1-t^{2}\right)^{\mu-1 / 2}, \quad \mu>-1 / 2 .
$$

Indeed, one has

$$
\rho(x)=\left(\frac{2}{b-a}\right)^{2 \mu-1}(b-x)^{\mu-1 / 2}(x-a)^{\mu-1 / 2} .
$$

Hence, from (6.1)

$$
\begin{aligned}
I(f) & =\int_{-1}^{1} f(t) \sigma(t) \mathrm{d} t=\int_{a}^{b} g(x) \rho(x) \mathrm{d} x \\
& =\left(\frac{2}{\sqrt{b}+\sqrt{a}}\right)^{2 \mu}\left(\frac{1}{\sqrt{b}-\sqrt{a}}\right) \int_{a}^{b} G(x) \omega(x) \mathrm{d} x,
\end{aligned}
$$

where $\omega(x)=(\sqrt{b}-\sqrt{a})^{1-2 \mu} x^{-\mu}\left[(b-x)^{\mu-1 / 2}(x-a)^{\mu-1 / 2}\right]$ and $G(x)=x^{\mu} g(x)$.

As we have seen in Sections 4 and 5 , the nodes and weights for the L-orthogonal quadrature formulas corresponding to $\omega$ are easily expressed in terms of the nodes $\left\{t_{j, n}\right\}_{j=1}^{n}$ and the weights $\left\{A_{j, n}\right\}_{j=1}^{n}$ of the Gauss-Christoffel formulas for $\sigma$. So, no extra computational effort needs to be done.

As for numerical experiments, we will concentrate on the weight function $\sigma \equiv 1$ on $(-1,1)$, i.e., $\mu=1 / 2$ in (6.2), so that

$$
I(f)=\int_{-1}^{1} f(t) \mathrm{d} t=\left(\frac{2}{b-a}\right) \int_{a}^{b} G(x) \frac{\mathrm{d} x}{\sqrt{x}},
$$

with $G(x)=\sqrt{x} f(h(x))$. Thus, the integral $I(f)$ in $(6.3)$ will be estimated by the $n$-point Gauss-Legendre formula; the integral in the right hand side of (6.3), by the corresponding $n$-point L-orthogonal formula for the weight function $x^{-1 / 2}$.

In the examples below, we compute integrals of the form

$$
I\left(f_{i}\right)=\int_{-1}^{1} f_{i}(t) \mathrm{d} t=\int_{-1}^{1} \frac{g_{i}(t)}{(t+1.01)^{\nu}} \mathrm{d} t
$$

taking $a=0.01$. Different choices of $g_{i}$ and $\nu$ are shown in Table 1, The graphics represent the relative errors, as a function of the number of nodes, both in the Gaussian (Gauss-Legendre) formula and in the L-orthogonal quadrature formula, when applied to both sides in (6.3).

TABLE 1.

\begin{tabular}{|c|c|c|}
\hline$i$ & $\nu$ & $g_{i}$ \\
\hline 1 & 3 & $\exp (t)$ \\
2 & $5 / 2$ & $\sin (t)$ \\
3 & 3 & $\exp \left((t+1.01)^{-1 / 2}\right)$ \\
4 & 3 & $\log (t+1.01)$ \\
\hline
\end{tabular}




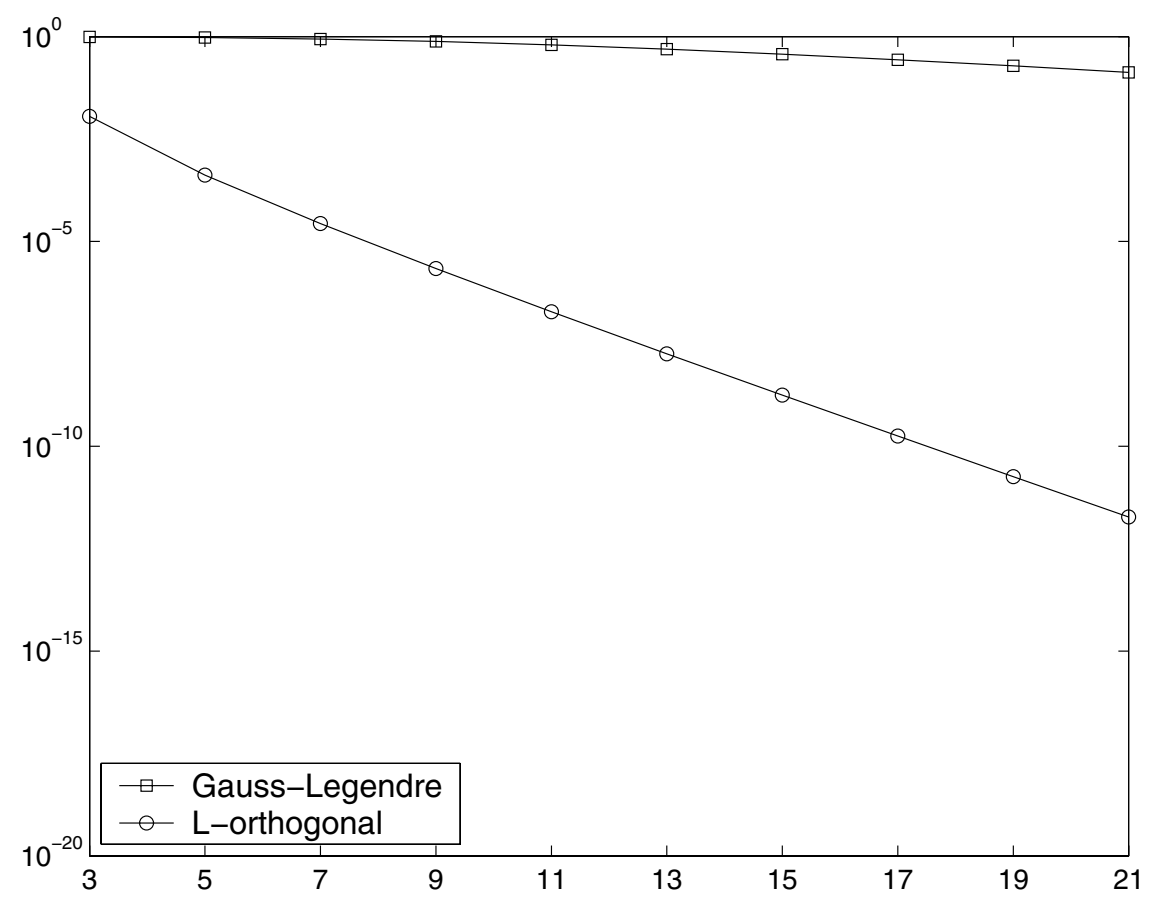

Figure 1. Relative error for $I\left(f_{1}\right)$

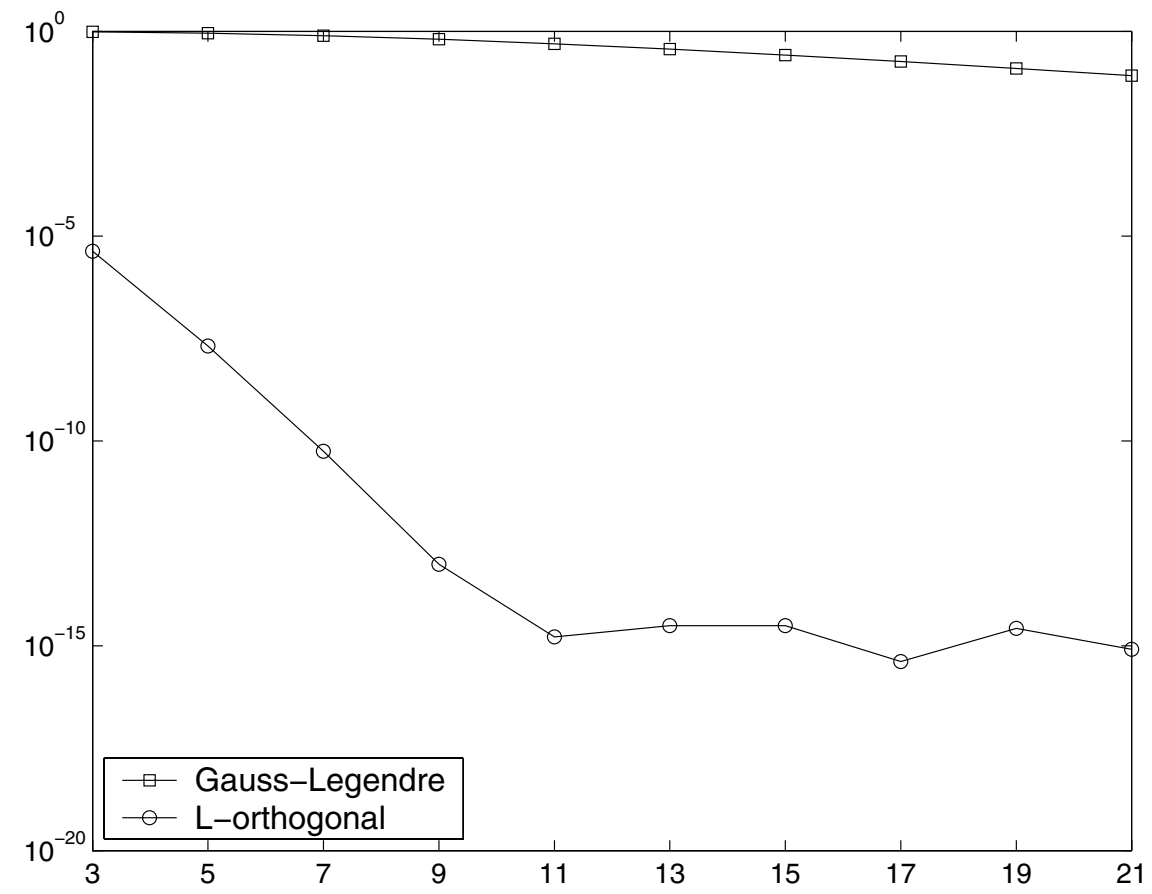

Figure 2. Relative error for $I\left(f_{2}\right)$ 


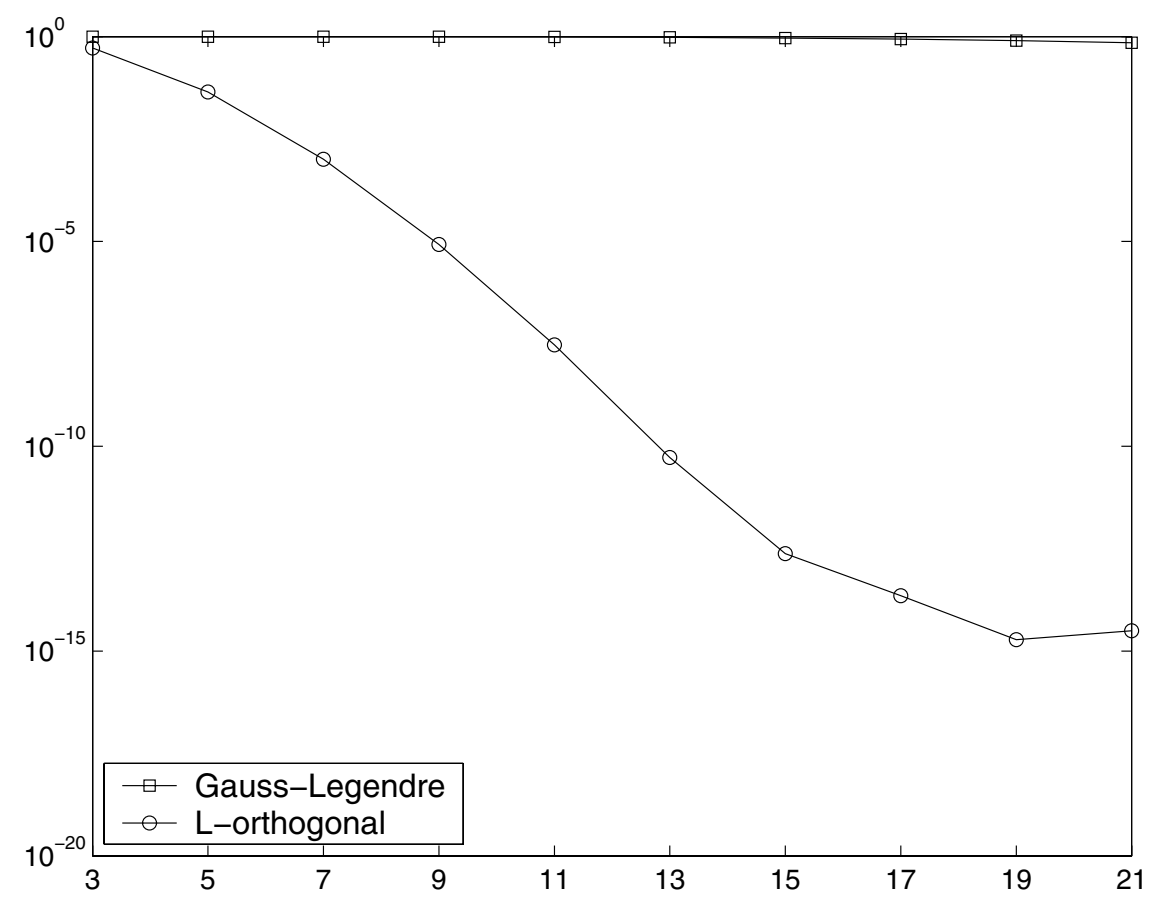

Figure 3. Relative error for $I\left(f_{3}\right)$

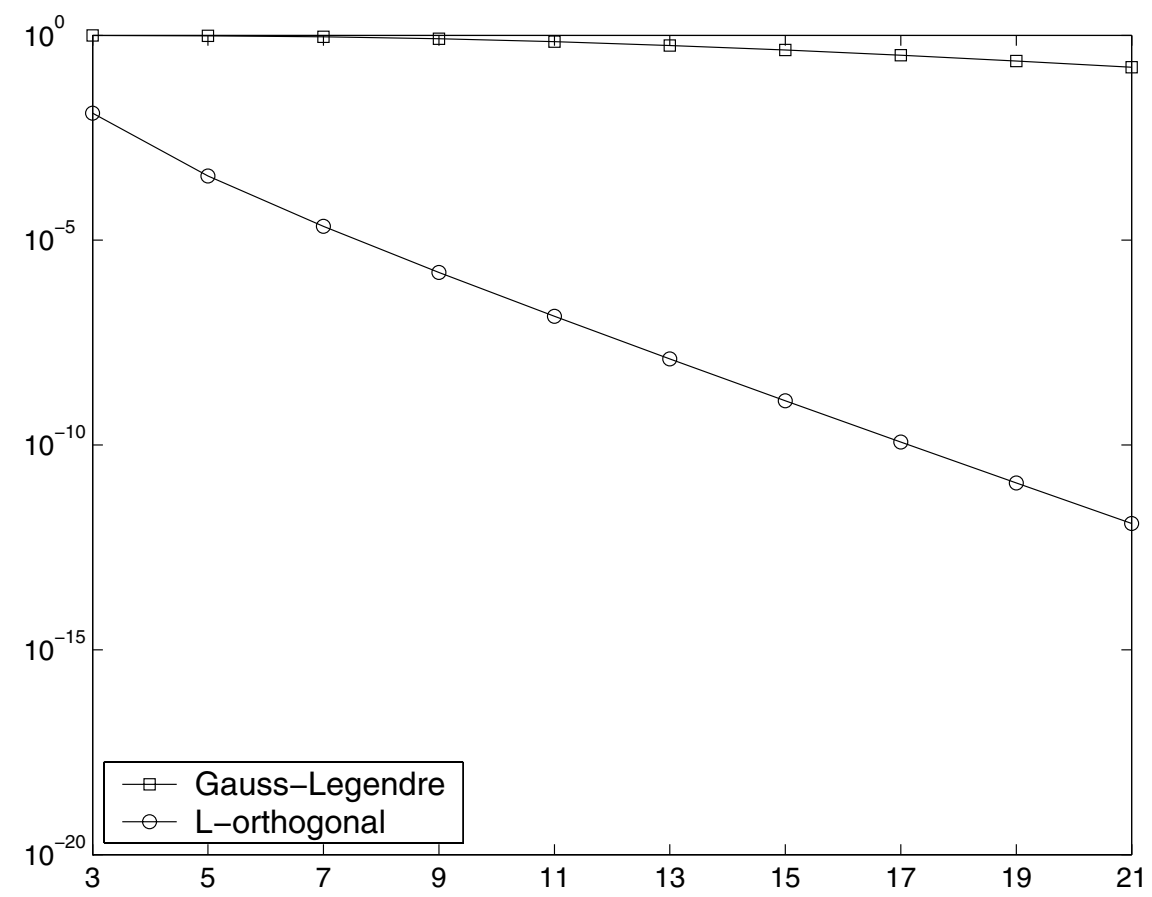

Figure 4. Relative error for $I\left(f_{4}\right)$ 
As could be expected from Figures 14, one sees that the convergence of the Gaussian formulas is extremely slow because of the presence of a multiple pole near the interval of integration. On the other hand, our L-orthogonal formulas provide excellent numerical results even when the integrand $f$ presents some kind of extra singularities, as in functions $f_{3}$ and $f_{4}$.

\section{AN APPLICATION TO INTERPOLATORY RULES}

As is known, trying to estimate a weighted integral $\int_{a}^{b} f(x) \mu(x) \mathrm{d} x$ by means of a classical Gaussian rule could require a long computational process if $\mu$ is not a standard weight anymore (see, e.g., [10] for details). Even more, Gaussian rules could become meaningless if $\mu$ is not a proper weight function $(\mu(x)>0$, a.e. on $(a, b))$, since now $\mu$ does not give rise, in general, to an inner product and nothing can be assured about the degree of the orthogonal polynomials and the location of their zeros. These drawbacks have motivated a possible alternative, the introduction of the so-called polynomial interpolatory rules, where for each $n$, distinct nodes $\left\{x_{j, n}\right\}_{j=1}^{n}$ in $(a, b)$ are given in advance and coefficients $\left\{A_{j, n}\right\}_{j=1}^{n}$ are deduced by imposing that

$$
\int_{a}^{b} f(x) \mu(x) \mathrm{d} x=\sum_{j=1}^{n} A_{j, n} f\left(x_{j, n}\right), \quad \forall f \in \Pi_{n-1},
$$

provided that the integrals $\int_{a}^{b} x^{k} \mu(x) \mathrm{d} x$ are easily computable, for each nonnegative integer $k$. Hence, the selection of the nodes turns out to be a crucial task in order to guarantee both convergence, and a reasonably good accuracy in a class of functions as large as possible. In this respect, the usage of zeros of orthogonal polynomials with respect to an auxiliary weight function on $(a, b)$ has become an extensively studied strategy, giving rise to excellent numerical results (see [11] and references therein). In a similar way, some of the present authors have considered in the last years, from a theoretical point of view, interpolatory rules now based upon Laurent polynomials (L-interpolatory rules); that is, given the family of distinct nodes $\left\{x_{j, n}\right\}_{j=1}^{n}$ on $(a, b)(0 \leq a<b \leq \infty)$, the coefficients $\left\{A_{j, n}\right\}_{j=1}^{n}$ are to be determined from

$$
\begin{aligned}
I_{\mu}(L) & =\int_{a}^{b} L(x) \mu(x) \mathrm{d} x \\
& =\sum_{j=1}^{n} A_{j, n} L\left(x_{j, n}\right)=I_{n}(L), \forall L \in \mathcal{L}_{n-1}=\Delta_{-p(n-1), q(n-1)},
\end{aligned}
$$

where, as usual, $\{p(n)\}_{n \geq 0}$ denotes a nondecreasing sequence of nonnegative integers such that $s(n)=p(n)-p(n-1) \in\{0,1\}$ and $q(n)=n-p(n)$. Here again, the crucial point is an adequate choice of the nodes. The term interpolatory is due to the property

$$
I_{n}(f)=I_{\mu}\left(L_{n}(f, \cdot)\right),
$$

with $L_{n}(f, \cdot) \in \mathcal{L}_{n-1}$ interpolating $f$ at the nodes $\left\{x_{j, n}\right\}_{j=1}^{n}$ (for interpolatory rules based upon rational functions, see [12]). The theoretical background supporting this kind of rules is the following. 
Theorem 7.1. Let $\mu$ be an $\mathrm{L}_{1}$-integrable function on $(a, b)(0 \leq a<b \leq \infty)$, and let $\omega$ be a strong weight function on $(a, b)$ such that

$$
\int_{a}^{b} \frac{|\mu(x)|^{2}}{\omega(x)} \mathrm{d} x<\infty .
$$

Let $\left\{R_{n}\right\}_{n \geq 0}$ be a sequence of orthogonal Laurent polynomials with respect to $\omega$ and the ordering induced by a given sequence $\{p(n)\}_{n \geq 0}$, and let $\left\{x_{j, n}\right\}_{j=1}^{n}$ be the zeros of $R_{n}$. For each $n=1,2, \ldots$, set $I_{n}(f)=\sum_{j=1}^{n} A_{j, n} f\left(x_{j, n}\right)$ such that $I_{n}(f)=I_{\mu}(f)$, $\forall f \in \mathcal{L}_{n-1}$. Then, it holds that

i) $\lim _{n \rightarrow \infty} I_{n}(f)=I_{\mu}(f)$, and

ii) $\lim _{n \rightarrow \infty} \sum_{j=1}^{n}\left|A_{j, n}\right| f\left(x_{j, n}\right)=\int_{a}^{b} f(x)|\mu(x)| \mathrm{d} x$, for any Riemann-Stieltjes integrable function with respect to $\omega$.

Remark 7.2. When dealing with a finite interval, a proof of this theorem for the polynomial case, i.e., $p(n)=0$ for each $n$, can be found in the excellent paper by Sloan and Smith [32, as a culmination of a long series of previous works. This result was extended to the Laurent situation in [4]. When concerning the interval $(0, \infty)$, see 23 for the polynomial case and [3] for the Laurent one. Here, it should be indicated that in this case, extra conditions on the moments $c_{k}=\int_{0}^{\infty} x^{k} \omega(x) \mathrm{d} x$ are required in order to guarantee convergence (see $[3]$ for details).

Remark 7.3. From ii) in Theorem 7.1, it follows that there exists a positive constant $M$, independent of $n$, such that

$$
\sum_{j=1}^{n}\left|A_{j, n}\right|<M, \quad n=1,2, \ldots
$$

Hence, the stability of the quadrature process is guaranteed.

On the other hand, up to now, the theoretical results obtained for these Linterpolatory rules have never been tested numerically, and this is precisely the main aim of this section. Thus for the computation of these rules, it seems clear that the most desirable situation could be to have at our disposal auxiliary weights $\omega$ satisfying (7.1) and whose family of orthogonal Laurent polynomials is explicitly known. In this respect, the examples given in Section 5 provide us with a list of possible good candidates by showing that the zeros of orthogonal Laurent polynomials with respect to some of these weight functions give rise to suitable L-interpolatory rules.

TABLE 2 .

\begin{tabular}{|c|c|c|}
\hline$i$ & $f_{i}$ & $r_{i}$ \\
\hline 1 & $\exp (x-1.01)$ & $x^{-1}$ \\
2 & $\exp \left(x^{-1 / 2}\right)$ & $x^{-1}$ \\
3 & $\log (x)$ & $x^{-1}$ \\
4 & $\exp (1.01-x)$ & $7 \pi(x-1.01)$ \\
\hline
\end{tabular}




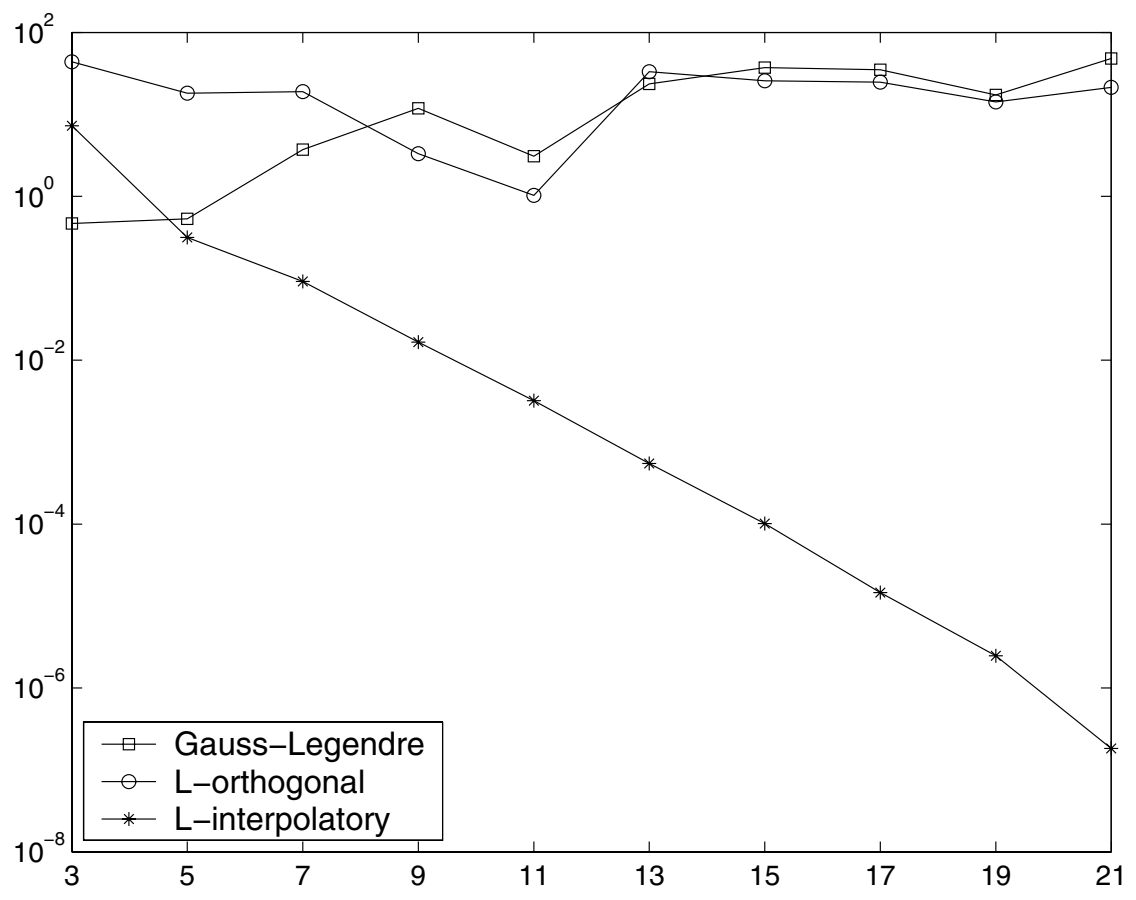

FiguRE 5. Relative error for $I\left(f_{1}\right)$

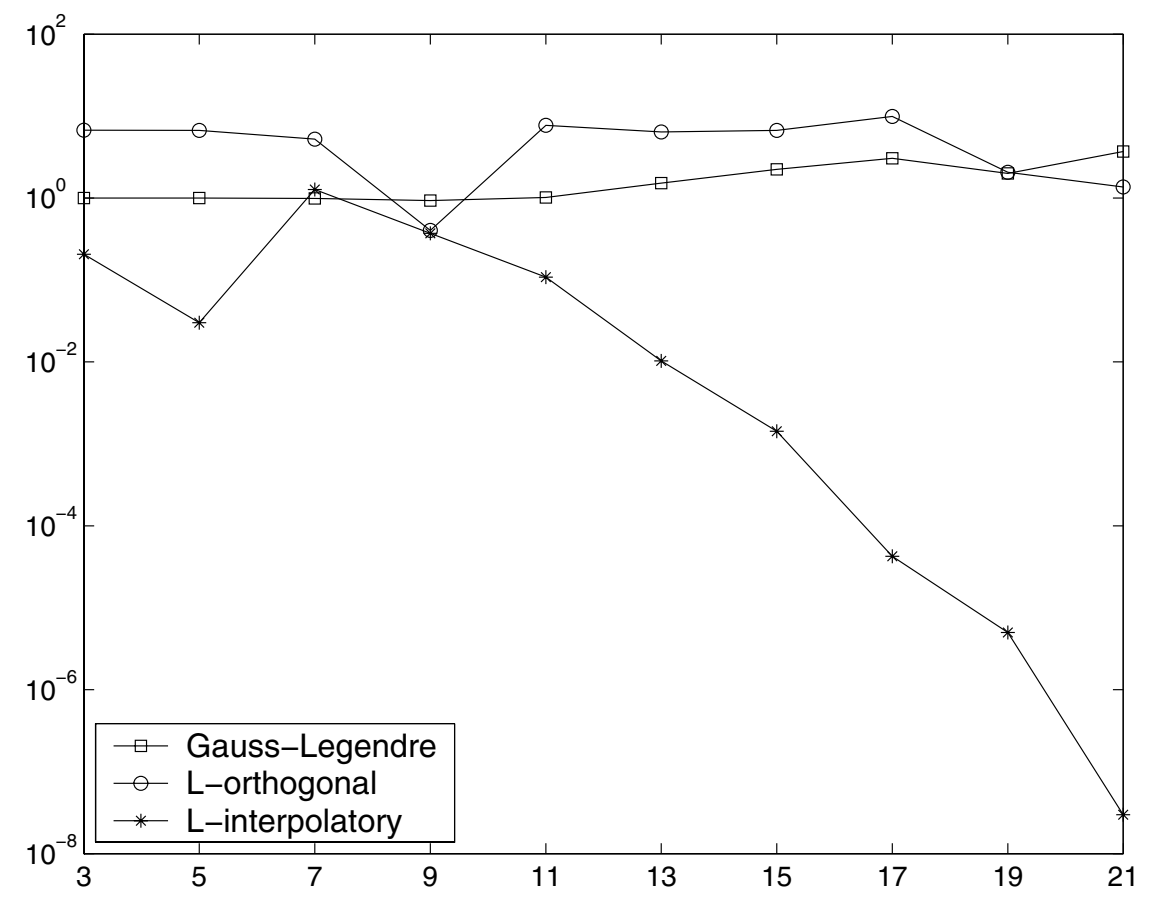

Figure 6. Relative error for $I\left(f_{2}\right)$ 


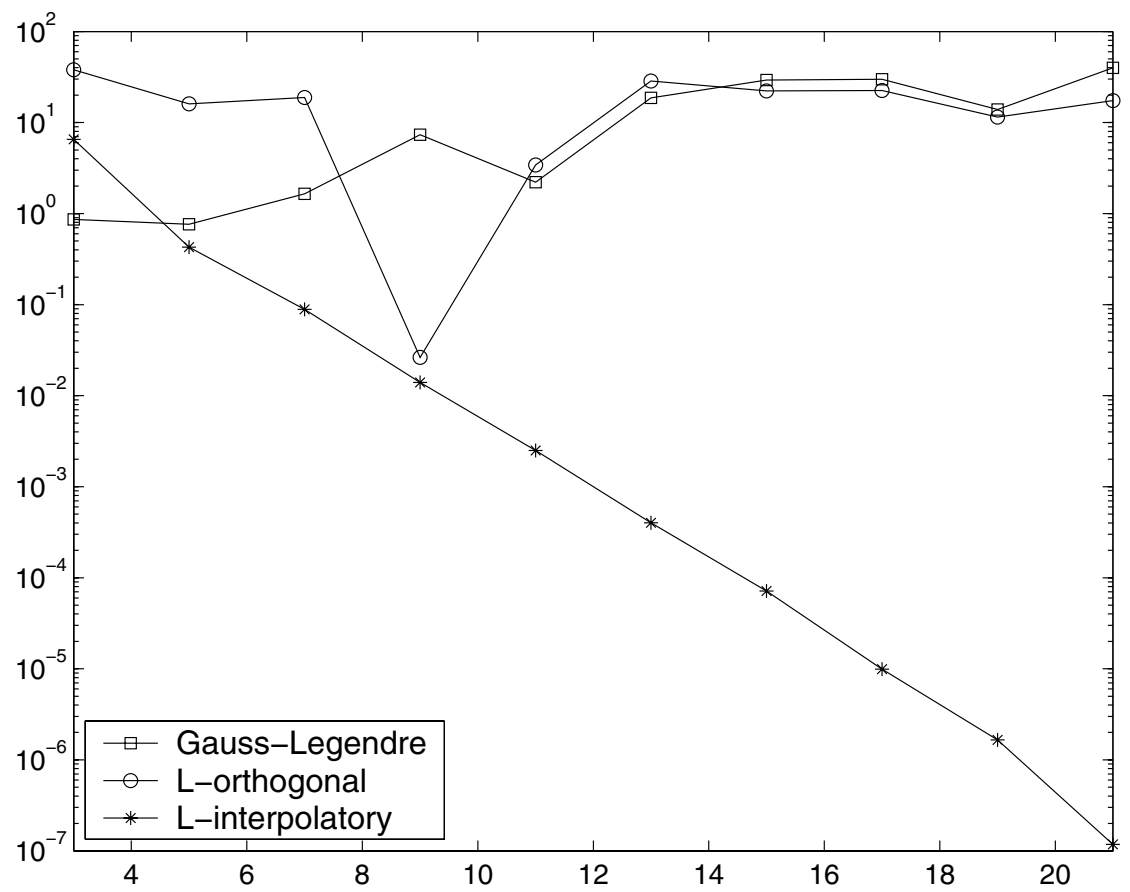

FiguRE 7. Relative error for $I\left(f_{3}\right)$

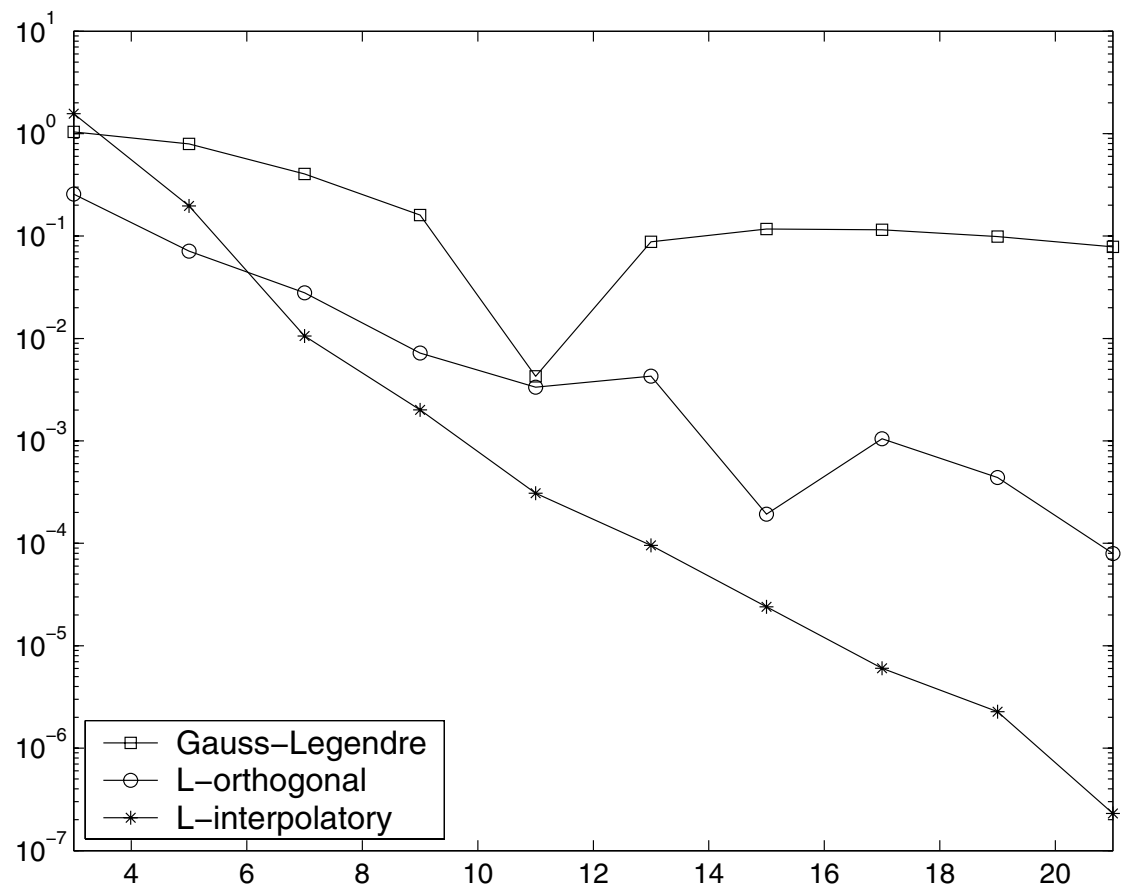

Figure 8. Relative error for $I\left(f_{4}\right)$ 
For our purposes, we will limit ourselves to the ordering induced by $p(n)=$ $E[(n+1) / 2]$ and integrals of the form

$$
I(f)=\int_{a}^{b} f(x) \mu(x) \mathrm{d} x,
$$

with $\mu(x)=x^{-1 / 2} \sin (r(x))$ ( $r$ is a polynomial or rational function), $0<a<$ $b<\infty$ and $f$ possessing possible singularities at the origin. Setting $I(f)=$

$\int_{a}^{b} F(x) x^{-1 / 2} \mathrm{~d} x$, we will also compute this last integral through an $n$-point Lorthogonal quadrature formula with respect to the weight function $\omega(x)=x^{-1 / 2}$. Now the numerical results are not, in general, as good as in Section 6 because of the oscillating factor $\sin (r(x))$, as can be seen from the figures. However, when estimating $I(f)$ in (7.2) by an $n$-point L-interpolatory rule taking as nodes the same ones as in the L-orthogonal formula (that is, the zeros of the $n$th orthogonal L-polynomial for $\omega(x)=x^{-1 / 2}$, which are easily expressed in terms of the zeros of Legendre polynomials), the numerical results are highly improved as displayed in the figures. Again we see that the computational cost is quite low.

Finally, observe that the fundamental condition (7.1)

$$
\int_{a}^{b} \frac{|\mu(x)|^{2}}{\omega(x)} \mathrm{d} x=\int_{a}^{b} \frac{|\sin (r(x))|^{2}}{\sqrt{x}} \mathrm{~d} x<\infty
$$

trivially holds.

The figures, as in Section 6, show the graphics of the relative error for the three rules proposed as a function of the number of nodes $n$. In all of the examples given we have taken $a=0.01, b=2.01$ and the integrals are of the form $I(f)=$ $\int_{a}^{b} f_{i}(x) \mu_{i}(x) \mathrm{d} x=\int_{a}^{b} f_{i}(x) x^{-1 / 2} \sin \left(r_{i}(x)\right) \mathrm{d} x$, with the functions $f_{i}$ and $r_{i}$ given in Table 2 for $i=1,2,3,4$. As seen from Figures 5 , the numerical effectiveness of our L-interpolatory rules is clearly displayed.

\section{Conclusions}

Gaussian formulas based upon usual polynomials have become an important topic within the field of numerical integration because of their numerical power and effectiveness, especially when dealing with smooth integrands. At the same time, these quadrature formulas have received much attention in the last decades as a result of their connection with the theory of orthogonal polynomials and related topics as Padé approximants. On the other hand, as pointed out in Section 1, quadrature formulas exactly integrating Laurent polynomials appeared as a working tool related to the solution of the so-called strong moment problems. During the last years, some of the present authors have considered such quadratures from the optic of a numerical integration approach, carrying out a series of numerical experiments (8], 2], 3]) and emphasizing their intimate relation to the theory of orthogonal Laurent polynomials and two-point Padé approximants. In this paper, we have intended to follow this line so that some results as, e.g., Proposition 4.5 which could have been deduced from the works by Ranga and collaborators (see, e.g., 30]), have now been revisited starting from the theory of orthogonal Laurent polynomials. In our opinion this approach seems more natural and appropriate for our final purpose, which is the approximate calculation of an integral. Nevertheless, we also think that much more work should be done in the future in order, for instance, to characterize, if possible, the L-orthogonal formulas in terms of the classical Gaussian ones for 
other orderings different from the one induced by $p(n)=\mathrm{E}[(n+1) / 2]$ as done in this paper. Here, finding an appropriate transformation like (4.2), seems to be the crucial point.

\section{REFERENCES}

1. A. Bultheel, P. González-Vera, E. Hendriksen and O. Njåstad, Orthogonal rational functions, Cambridge Monographs on Applied and Computational Mathematics, 5. Cambridge University Press, Cambridge, 1999. MR1676258(2000c:33001)

2. A. Bultheel, C. Díaz-Mendoza, P. González-Vera and R. Orive, On the convergence of certain Gauss-type quadrature formulas for unbounded intervals, Math. Comp. 69, no. 230, (2000) 721-747. MR1651743 (2000i:65034)

3. A. Bultheel, C. Díaz-Mendoza, P. González-Vera and R. Orive, Orthogonal Laurent polynomials and quadrature formulas for unbounded intervals: II Interpolatory rules, Preprint, 2003.

4. A. Bultheel, C. Díaz-Mendoza, P. González-Vera and R. Orive, Quadrature on the half line and two-point Padé approximants to Stieltjes functions. Part II. Convergence, J. Comput. Appl. Math. 77 (1997), 53-76. MR1440004(98a:41023)

5. L. Cochran and and S. Clement Cooper, Orthogonal Laurent polynomials on the real line, in: Continued fractions and orthogonal functions (Loen, 1992), eds S. Clement Cooper and W. J. Thron, Lecture Notes in Pure and Appl. Math., 154, Dekker, New York, 1994, pp. 47-100. MR.1263248 (95b:42024)

6. R. Cruz-Barroso and P. González-Vera, Orthogonal Laurent polynomials and quadratures on the unit circle and the real half-line, to appear in Electron. Trans. Numer. Anal., 2003.

7. C. Díaz-Mendoza, P. González-Vera and R. Orive, Padé approximats and quadratures related to certain strong distributions, J. Comput. Appl. Math. 133 (2001), no. 1-2, 315-329. MR.1858290 (2002g:41025)

8. C. Díaz-Mendoza, P. González-Vera, and M. Jiménez Paiz, Strong Stieltjes distributions and orthogonal Laurent polynomials with applications to quadratures and Padé approximation, Preprint 2003.

9. W. Gautschi, On generating orthogonal polynomials. SIAM J. Sci. Statist. Comput. 3 (1982), no. 3, 289-317. MR0667829 (84e:65022)

10. W. Gautschi, Numerical Analysis. An introduction, Birkhäuser Boston, 1997. MR 1454125 (98d:65001)

11. P. González-Vera, G. López Lagomasino, R. Orive and J.C. Santos, On the convergence of quadrature formulas for complex weight functions, J. Math. Anal. Appl. 189 (1995), 514-532. MR.1312059 (95k:65027)

12. P. González-Vera, M. Jiménez Paiz, R. Orive and G. López Lagomasino, On the convergence of quadrature formulas connected with multipoint Padé-type approximation, J. Math. Anal. Appl. 202 (1996), 747-775. MR1408352 (97e:41066)

13. B. A. Hagler, A transformation of orthogonal polynomial sequences into orthogonal Laurent polynomial sequences, $\mathrm{Ph}$. D. Thesis, University of Colorado, 1997.

14. B. A. Hagler, Formulas for the moments of some strong moment distributions, in: Orthogonal functions, moment theory, and continued fractions (Campinas, 1996), eds. W.B. Jones and S.A. Ranga, Lecture Notes in Pure and Appl. Math., 199, Dekker, New York, 1998, pp. 179-186. MR1655662 (2000a:44006)

15. B. A. Hagler, W.B. Jones and W.J. Thron, Orthogonal Laurent polynomials of Jacobi, Hermite, and Laguerre types, in: Orthogonal functions, moment theory, and continued fractions (Campinas, 1996), eds. W.B. Jones and S.A. Ranga, Lecture Notes in Pure and Appl. Math., 199, Dekker, New York, 1998, pp. 187-208. MR.1655663 (2000a:33013)

16. E. Hendriksen, A characterization of classical orthogonal Laurent polynomials, Nederl. Akad. Wetensch. Indag. Math. 50, no. 2 (1988), 165-180. MR0952513 (89h:33014)

17. W.B. Jones, W.J. Thron and H. Waadeland, A strong Stieltjes moment problem, Trans. Amer. Math. Soc. 261, no. 2 (1980), 503-528. MR0580900 (81j:30055)

18. W.B. Jones and W.J. Thron, Orthogonal Laurent polynomials and Gaussian quadrature, in: Quantum Mechanics in Mathematics, Chemistry and Physics, eds. K.e E. Gustafson and W. P. Reinhardt, Plenum Press N.Y., 1981, pp. 449-455. 
19. W.B. Jones, O. Njåstad and W.J. Thron, Two-point Padé expansions for a family of analytic functions, J. Comput. Appl. Math. 9, no. 2 (1983), 105-123. MR0709210 (84j:30057)

20. W.B. Jones and O. Njåstad, Orthogonal Laurent polynomials and strong moment theory: a survey, J. Comput. Appl. Math. 105, no. 1-2 (1999), 51-91. MR1690578 (2000d:30054)

21. Klaus-Jürgen Förster and K. Petras, On estimates for the weights in Gaussian quadrature in the ultraspherical case, Math. Comp. 55, no. 191, (1990), 243-264. MR.1023758 (91d:65043)

22. O. Njåstad and W.J. Thron, The theory of sequences of orthogonal L-polynomials, Det Kong. Norske Vid. Selsk. 1 (1983), 54-91.

23. A.H. Opie, I.H. Sloan, and W.E. Smith, Product integration over infinite intervals I. Rules based on the zeros of Hermite polynomials, Math. Comp., v. 40 , no. 162 (1983), 519-535. MR.0689468 (85a:65047)

24. K. Petras, An asymptotic expansion for the weights of Gaussian quadrature formulae, Acta Math. Hungar. 70, no. 1-2, (1996), 89-100. MR1361463 (96h:41034)

25. J. Sánchez-Ruiz, Information entropy of Gegenbauer polynomials and Gaussian quadrature, J. Phys. A 36, no. 17, (2003), 4857-4865. MR1984014 (2004f:33025)

26. A. Sri Ranga and J.H. McCabe, On the extensions of some classical distributions, Proc. Edinb. Math. Soc., II. Ser. 34, no.1, (1991), 19-29. MR.1093173 (92b:30003)

27. A. Sri Ranga, Another quadrature rule of highest algebraic degree of precision, Numer. Math. 68, no.2, (1994), 283-294. MR.1283343 (95c:65047)

28. A. Sri Ranga, Symmetric orthogonal polynomials and the associated orthogonal L-polynomials, Proc. Am. Math. Soc. 123, no.10, (1995), 3135-3141. MR1291791 (95m:42035)

29. A. Sri Ranga, E.X.L. de Andrade and J.H. McCabe, Some consequences of a symmetry in strong distributions, J. Math. Anal. Appl. 193, no.1, (1995), 158-168. MR1338505(97a:42022)

30. A. Sri Ranga, E.X.L. de Andrade and G.M. Phillips, Associated symmetric quadrature rules, Appl. Numer. Math. 21, No.2, (1996) 175-183. MR1413592(97g:65061)

31. A. Sri Ranga, W. Van Assche, Blumenthal's theorem for Laurent orthogonal polynomials, J. Approx. Theory 117, no.2, (2002), 255-278. MR1903057 (2003g:42044)

32. Sloan, I. H. y Smith, W. E. Properties of interpolatory product integration rules, SIAM J. Numer. Anal. 19 (1982), 427-442. MR0650061 (83e:41032)

33. Gábor Szegö, Orthogonal polynomials, Fourth edition, American Mathematical Society, Colloquium Publications, Vol. XXIII, American Mathematical Society, Providence, R.I., 1975. MR0372517(51:8724)

Departamento de Análisis Matemático, Universidad de La Laguna 38271 La Laguna, Tenerife, Canary Islands, Spain

E-mail address: cjdiaz@ull.es

Departamento de Análisis Matemático, Universidad de La Laguna 38271 La Laguna, Tenerife, Canary Islands, Spain

E-mail address: pglez@ull.es

Departamento de Análisis Matemático, Universidad de La Laguna 38271 La Laguna, Tenerife, Canary Islands, Spain

E-mail address: mjimenez@ull.es

Centro de Docencia Superior en Ciencias Básicas, Campus Puerto Montt, Universidad Austral de Chile, Puerto Montt, Chile

E-mail address: fcala@uach.cl 